\title{
Lower limb in the preparticipation examination (PPE)
}

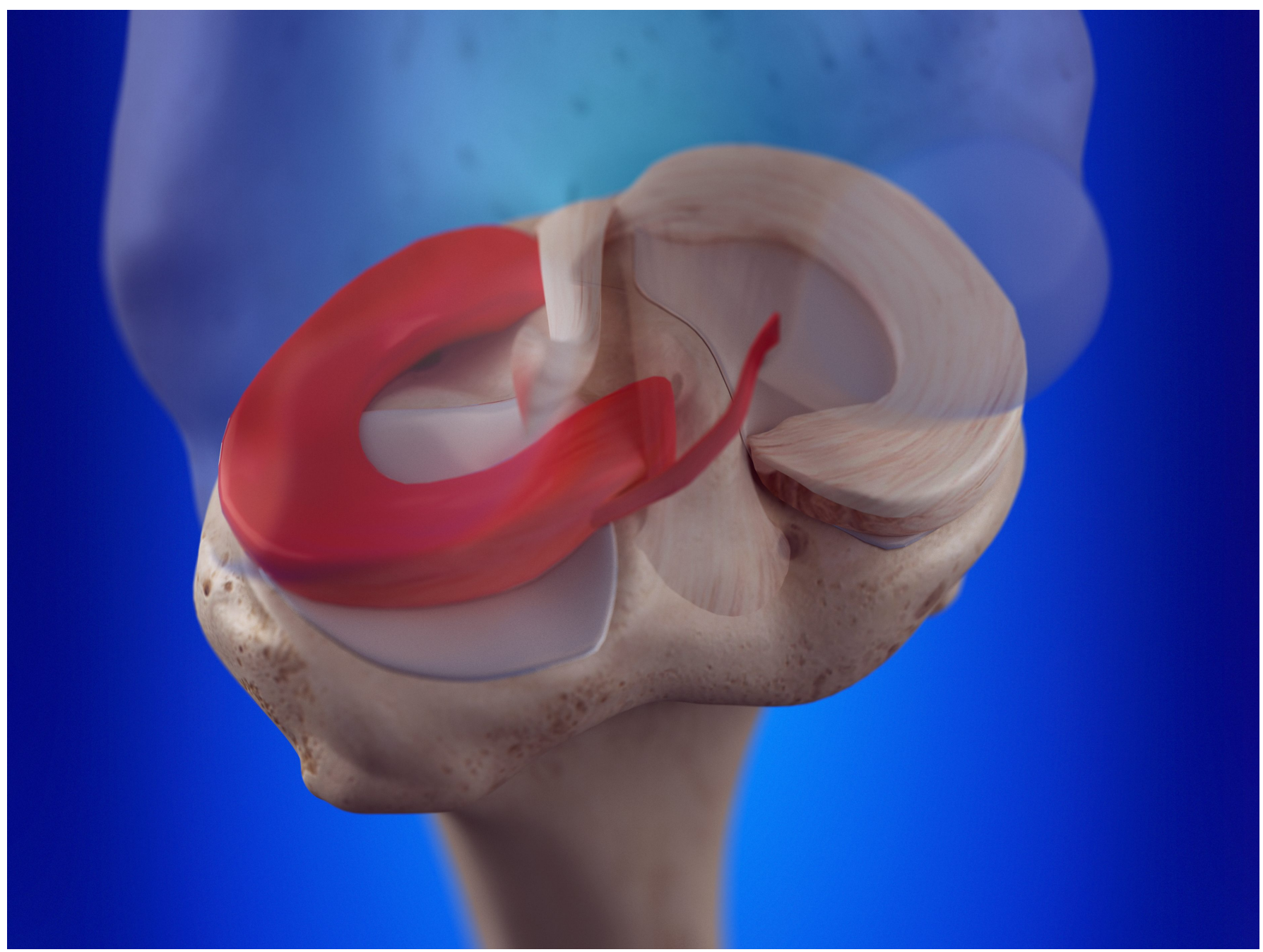

Die Untersuchung der unteren Extremität in der sportärztlichen Untersuchung (SPU)

Leumann $\mathrm{A}^{1,}{ }^{2}$, Schmid $\mathrm{AK}^{2},{ }^{4}$, Fischer $\mathrm{M}^{3}$, Fromm $\mathrm{L}^{5}$, Smirnov $\mathrm{E}^{6}$

${ }^{1}$ OrthoPraxis Leumann AG, Basel

${ }^{2}$ Labor für Biomechanik, Orthopädische Universitätsklinik, Universität Basel

${ }^{3}$ Physio Active Care GmbH, Basel 
${ }^{4}$ Institut für Rechtsmedizin, Universität Basel

${ }^{5}$ Gesundheitszentrum Unterengadin, Schuls/Scuol

${ }^{6}$ Orthopädische Klinik, Kantonsspital Baselland

\section{Abstract}

This article described the clinical examination of the lower extremity during a pre-participation screening in regard of sports ability, presence of injuries and musculoskeletal disorders as well as predisposing risk factors for injuries and prevention. It divided into global static and dynamic testing but also isolated analysis of joint function.

\section{Zusammenfassung}

Der vorliegende Artikel beschreibt einen möglichen Untersuchungsgang zur Beurteilung der unteren Extremität in einer sportärztlichen Untersuchung hinsichtlich der Sportfähigkeit, dem Vorhandensein von Verletzungen und Beschwerden und dem Vorliegen von prädisponierenden Faktoren bezüglich Verletzungsanfälligkeit und Prävention. Er gliedert sich dabei in eine kursorische statische und dynamische Gesamtanalyse und betrachtet zusätzlich die grossen Gelenke isoliert.

\section{Einleitung}

Beschwerden und Verletzungen der unteren Extremität sind sehr häufig. Am häufigsten entstehen Verletzungen dabei im Fussball und im Wintersport. Dabei sind bei frischen Verletzungen gemäss SUVAStatistik 2018 die Gelenke folgendermassen mitbetroffen: Hüfte 3\%, Knie 10\%, Sprunggelenk und Fuss 24\%. Für viele Verletzungen und Beschwerden gibt es a) einen Zusammenhang zur Sportart («Déformation professionelle») und b) einen Zusammenhang zur Trainingsgestaltung («zu viel ist ungesund»). Dabei können zusätzliche prädisponierende extrinsische (z.B. Ausrüstung, Witterung, Gegnerkontakt) und intrinsische (z.B. anatomische Fehlstellung, Gelenksbeweglichkeit, Kraft) Faktoren vorhanden sein. In der sportärztlichen Untersuchung geht es primär um die Adressierung von prädisponierenden Risikofaktoren im Sinne einer Verletzungs-Prävention und um die Früherkennung von Sportart- oder Verletzungs-bezogenen Folge- und Dauerschäden. Beides gehört natürlich genauso dazu, wenn sich ein/e Athlet/-in mit einer neuen Symptomatik oder frischen Verletzung beim Team- oder Sportarzt vorstellt. Für eine klinischen Untersuchung ist die Erfahrung des Untersuchers - zur Beurteilung, was normal ist entscheidend.

Zur Diagnosestellung gehört neben der körperlichen Untersuchung eine umfassende Anamnese und gegebenenfalls weiterführende Diagnostik (Röntgen, Ultraschall, MRI, Labor, EMG usw.). Nur aus einer korrekten Diagnosestellung kann eine sinnvolle Therapie und Rehabilitationsplanung erfolgen. Für Letztere muss in der Sportmedizin oft Therapeut, Trainer und Betreuer miteinbezogen werden. Für die sportärztliche Untersuchung der unteren Extremität ist es dabei zentral, sich am Konzept «das Gelenk als Organ» zu orientieren und sich nicht nur auf einen möglichen Schmerzpunkt oder das reibungsfreie Gleiten einer Gelenksfläche zu fokussieren. Betrachtet man das Gelenk als Organ, so befinden sich alle am Gelenk beteiligten Gewebe in einer Homöostase («Fliess-Gleichgewicht»). Der 
individuelle Steady-state wird durch andauernde Anpassungsmechanismen der Gewebe aufrechterhalten («Trainingseffekt») (Tab. 1).

Der vorliegende Artikel hat zum Zweck, die Untersuchung der unteren Extremität in der sportärztlichen Untersuchung zu beschreiben. Er orientiert sich dabei an obengenannten Vorgaben auf Grundlage der Mini C-Ex Unter-

suchungen der SGSM (https://sgsm.ch/weiter-fortbildung/weiterbildung/logbook) sowie der neu überarbeiteten SPU-Dokumente (Abb. 1; https://sgsm.ch/publikationen/spu-dokumente). Der Artikel kann nicht eine detaillierte orthopädische oder rheumatologische Untersuchung einer spezifischen Pathologie abdecken.
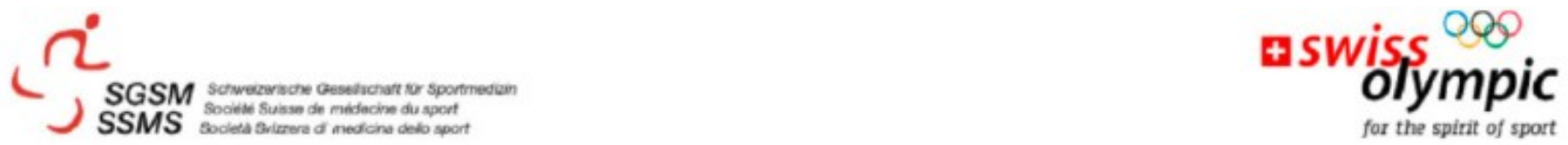

o.B. signifikanter Befund

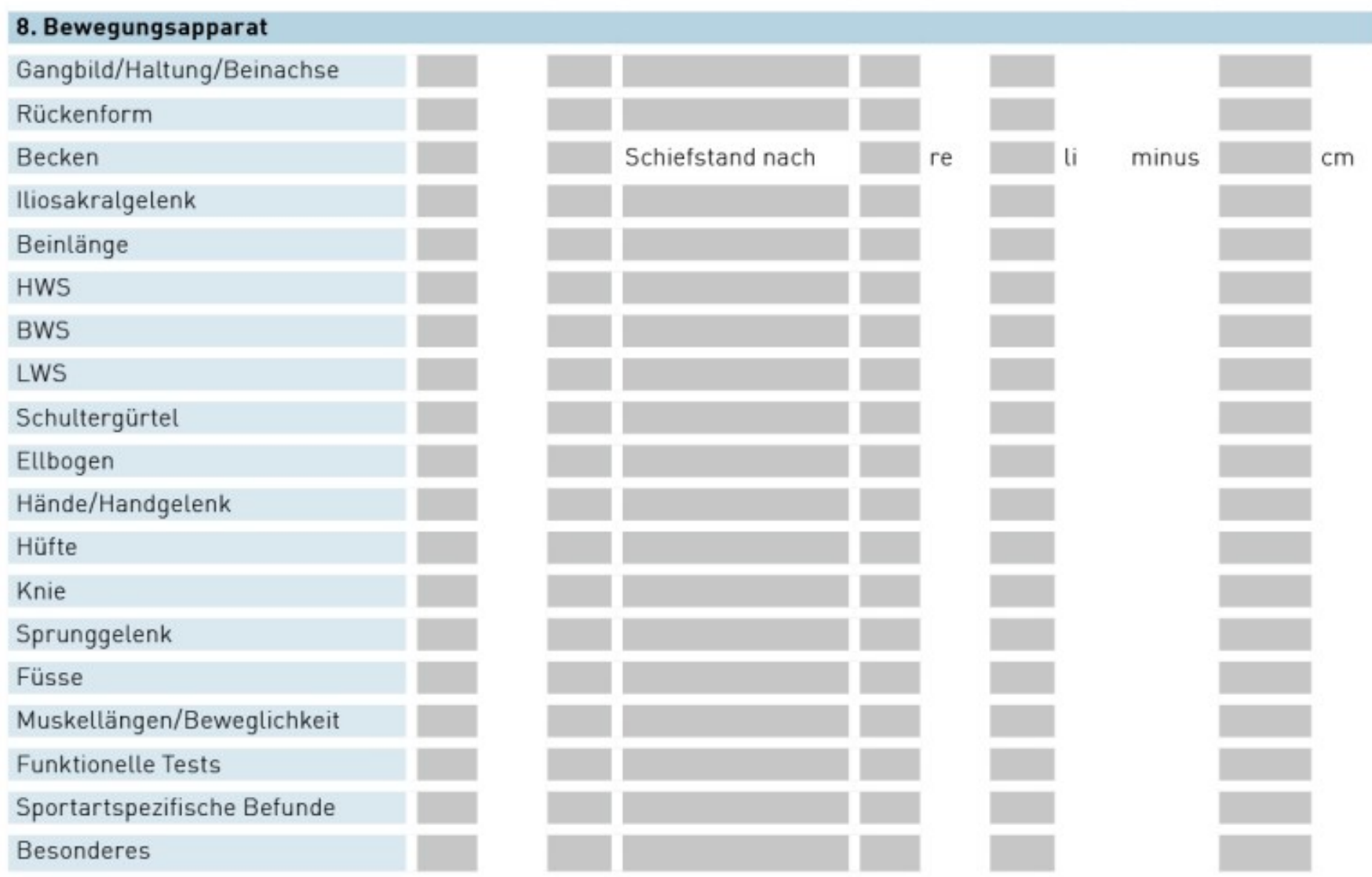




\begin{tabular}{|c|c|c|}
\hline Gewebe & Funktion & Überlastung \\
\hline Knochen & Skelett & Stressfraktur \\
\hline Knorpel & $\begin{array}{l}\text { Modulation und Dämpfung der Kraft- } \\
\text { übertragung im Gelenk }\end{array}$ & Degeneration, Volumenverlust \\
\hline Kapsel, Synovia & $\begin{array}{l}\text { Produktion der Gelenksflüssigkeit, } \\
\text { Auskleidung der Gelenkshöhle }\end{array}$ & Erguss \\
\hline Muskel & $\begin{array}{l}\text { Motor für die aktive Beweglichkeit des } \\
\text { Gelenks }\end{array}$ & Muskeldysbalance, -atrophie \\
\hline Sehnen & $\begin{array}{l}\text { Kraftübertragung vom Muskel auf den } \\
\text { Knochen }\end{array}$ & Tendinose, (Peri-)Tendinitis \\
\hline Ligamente & $\begin{array}{l}\text { Führung und Limitierung der Gelenks- } \\
\text { beweglichkeit }\end{array}$ & Instabilität \\
\hline Nerven & $\begin{array}{l}\text { Steuerung und Rückkoppelung der } \\
\text { Bewegung }\end{array}$ & Lokale Fatigue [?] \\
\hline Andere [z.B. Labrum, Meniskus] & $\begin{array}{l}\text { Gelenksspezifische Strukturen zur Verbes- } \\
\text { serung der Gelenkskongruenz und Gelenk- } \\
\text { funktion (Kraftübertragung. Propriozeption) }\end{array}$ & Degeneration, degenerative Läsion \\
\hline
\end{tabular}

\section{Globale Untersuchung der unteren Extremität}

\section{Statische Untersuchung im Stehen}

Die statische Untersuchung der unteren Extremität hat im Stehen zu erfolgen. Dabei können folgende Faktoren erfasst werden:

- Beckenstand: Ein Schiefstand wird zur verkürzten Seite angegeben (cm).

- Beinachse: Bei einer orthograden Beinachse berühren sich exakt die Epicondylen und die medialen Malleolen. Bei einem Genu varum kann der Condylenabstand $(\mathrm{cm})$ und bei einem Genu valgum der Malleolenabstand $(\mathrm{cm})$ angegeben werden.

- Torsionachsen (Untersuchung im Sitzen):

- Femurtorsion: Beurteilt die Achse des Schenkelhalses in Rotation zur Condylenachse am Knie. Sie verändert sich im Laufe des Wachstums (Abb. 2).

- Tibiatorsion: Beurteilt die (Aussen-)Rotation der Malleolengabel im Vergleich zur Achse des Tibiaplateaus (Abb. 3).

- Fussstellung:

- Rückfussachse: Analysiert die Stellung des Calcaneus im Verhältnis zur Tibiaachse von posterior (Winkelgrad). Als Norm gilt $0-10^{\circ}$ Valgus.

- $\square$ Mittelfussgewölbe: kann zu flach (planus; Knick-Senkfuss/Plattfuss) oder zu hoch (cavus; Hohlfuss) sein und kommt in der Regel in Kombination mit einer Fehlstellung des Rückfusses vor (Pes planovalgus - Pes cavovarus). Normal ist im lateralen Mittelfuss die Belastungszone einen Daumen breit. Bei der Rotationsstellung des Fusses muss unterschieden werden, ob es sich um eine Fussbezogene Fehlstellung (Adduktion - Abduktion aus dem Mittelfuss) handelt oder um eine Fehlstellung aus einer Rotationsfehlstellung des Beines (z.B. Tibiatorsion).

- Vorfuss: ein verbreitertes und abgeflachtes Quergewölbe entspricht einem Spreizfuss.

- Weitere Fusstypisierungen, z.B. griechischer Fuss [2. Zehe am längsten], ägyptischer Fuss [1. Zehe am längsten]) sind medizinisch nicht von Bedeutung. 


\section{Inspektion und Palpation}

Die Inspektion wie die Palpation beruhen auf den Grundlagen der Anatomie. Basierend auf ossären Landmarken können die einzelnen Strukturen ertastet werden. Diese Untersuchung ist insbesondere für das Knie (Abb. 4) und den Fuss (Abb. 5) sehr ergiebig, da sich viele Strukturen direkt unter der Haut palpieren lassen. Von Seiten des Sports ist in der Inspektion ein besonderes Augenmerk auf die Muskeltrophik zu werfen.

\section{Bewegungsumfang (Neutral-Null-Methode)}

Der Bewegungsumfang der Gelenke wird nach der Neutral-Null-Methode im Seitenvergleich beschrieben (Tab. 2). Dabei ist es wichtig, die Nullstellung zu kennen, sowie die Position der Nachbargelenke, um den Effekt von zweigelenkigen Muskeln zu neutralisieren (z.B. Triceps surae, Hamstrings, Rectus femoris).

\section{Neurologische Untersuchung}

Zur kursorischen Überprüfung der peripheren Nervenfunktion gehört die Untersuchung der Sensorik, der Motorik und der Reflexe. Hierbei ist die Orientierung einerseits segmental oder Dermatom bezogen, andererseits entsprechend den peripheren Nervenverläufen. Die Sensorik kann asensibel, hyposensibel oder hypersensibel sein, die Motorik wird nach dem MRC-Scale (Medical Research Council) eingeteilt: $\mathrm{M}^{5}$, normale Kontraktur gegen vollen Widerstand; $\mathrm{M}^{4}$, Muskelkraft ist reduziert, aber die Muskelkraft kann das Gelenk noch gegen Widerstand bewegen; $\mathrm{M}^{3}$, stärker reduzierte Muskelkraft, sodass der Muskel das Gelenk noch gegen die Schwerkraft, aber nicht mehr gegen Widerstand bewegen kann; $\mathrm{M}^{2}$, Bewegung ist nur noch möglich, wenn die Schwerkraft eliminiert wird; $\mathrm{M}^{1}$, es ist noch eine Muskelaktivität sichtbar, daraus allerdings resultiert keine Bewegung, $\mathrm{M}^{0}$, keine Muskelaktivität sichtbar.

\begin{tabular}{|c|c|c|c|}
\hline Gelenk & Range of Motion & Normalwerte (Winkelgrad) & Besonderes \\
\hline \multirow[t]{3}{*}{ Hüfte } & Flexion/Extension & $130-140 / 0 / 10-20^{\circ}$ & \\
\hline & Adduktion/Abduktion & $20-30 / 0 / 30-50^{\circ}$ & \\
\hline & Innenrotation/Aussenrotation & $40-50 / 0 / 30-45^{\circ}$ & $90^{\circ}$ flektierte Hüfte \\
\hline \multirow[t]{2}{*}{ Knie } & Flexion/Extension & $120-150 / 0 / 5-10^{\circ}$ & \\
\hline & Innenrotation/Aussenrotation & $10 / 0 / 25^{\circ}$ & $90^{\circ}$ flektiertes Knie \\
\hline Oberes Sprunggelenk & $\begin{array}{l}\text { Dorsalextension/Plantar- } \\
\text { flexion }\end{array}$ & $20-30 / 0 / 40-50^{\circ}$ & $90^{\circ}$ flektiertes Knie \\
\hline Unteres Sprunggelenk & Inversion/Eversion & $20 / 0 / 16^{\circ}$ & $\begin{array}{l}90^{\circ} \text { flektiertes Knie, } \\
\text { Oberes Sprunggelenk in } \\
\text { Neutralstellung }\end{array}$ \\
\hline Rückfuss & Pronation/Supination & $35 / 0 / 15^{\circ}$ & \\
\hline Grosszehengrundgelenk & $\begin{array}{l}\text { Dorsalextension/Plantar- } \\
\text { flexion }\end{array}$ & $75 / 0 / 45^{\circ}$ & \\
\hline
\end{tabular}




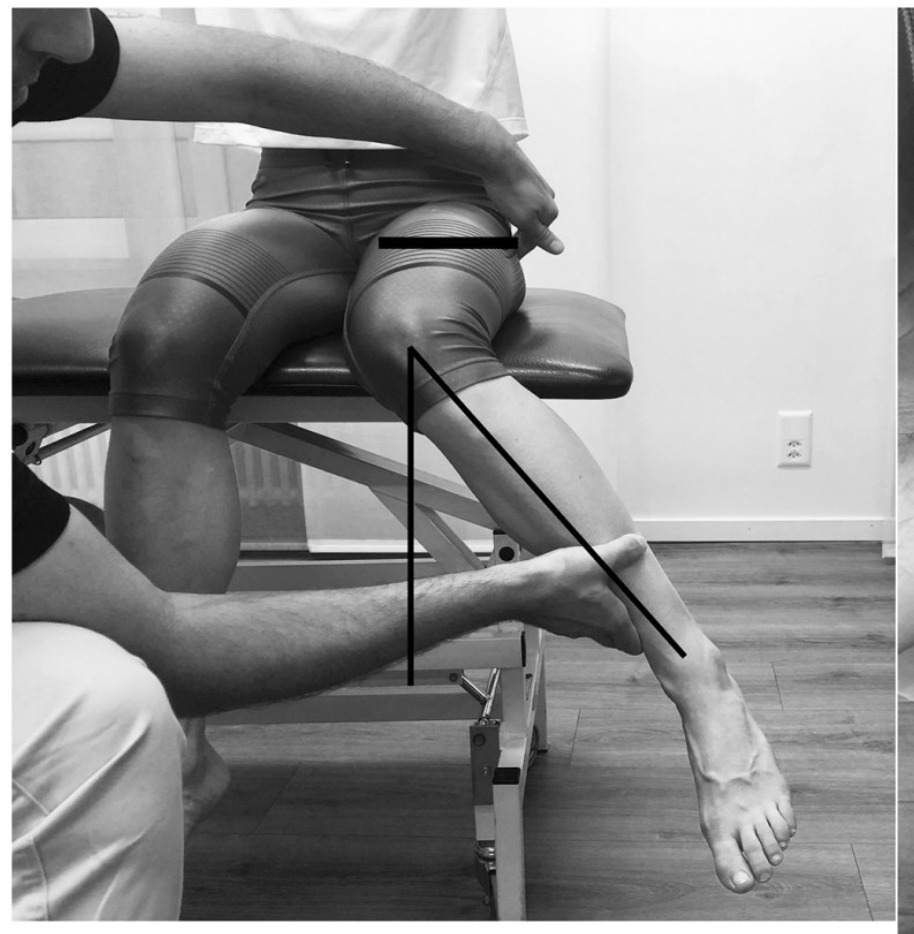

Abb. 2: Bestimmung der femoralen Antetorsion. Der Fuss wird so weit nach aussen gedreht, bis der Trochanter maior maximal weit aussen getastet werden kann und die Schenkelhalsachse horizontal ist. Der Winkel der Rotation der Tibiaachse aus der Vertikalen entspricht der femoralen Antetorsion.

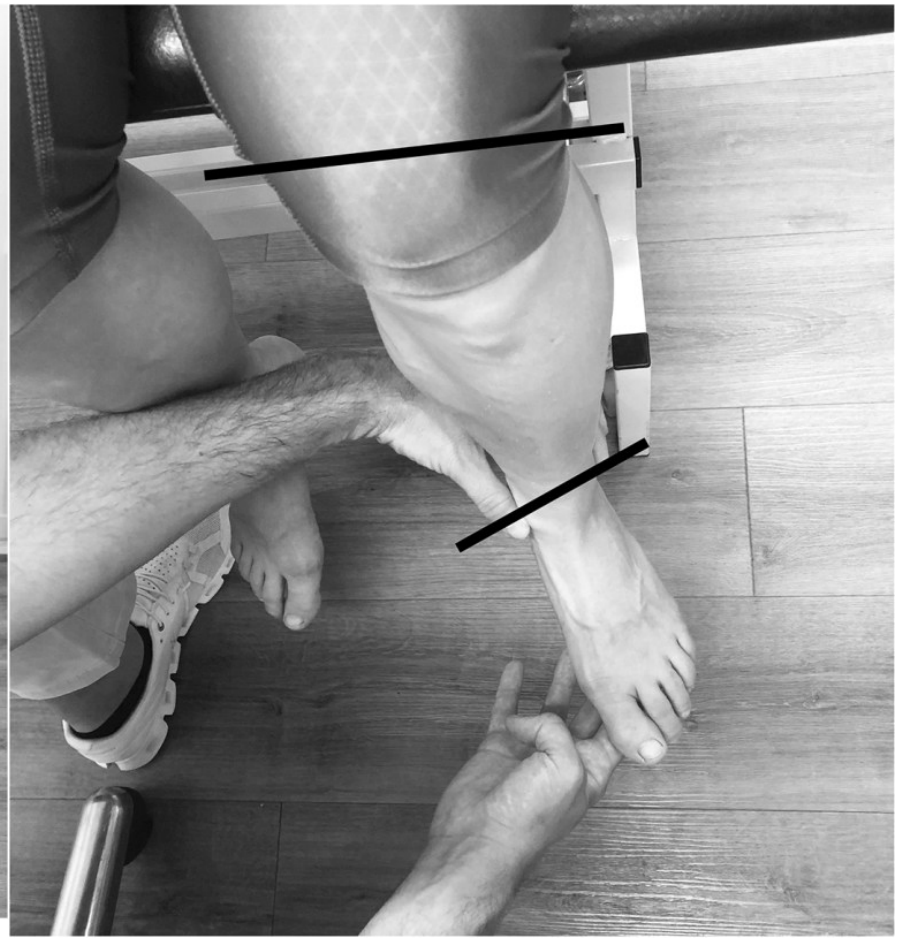

Abb. 3: Bestimmung der Tibiatorsion. In 90 Knieflexion wird die Knierotation neutralisiert. Die Abweichung der Achse durch die Malleolengabel entspricht der Tibiatorsion. 

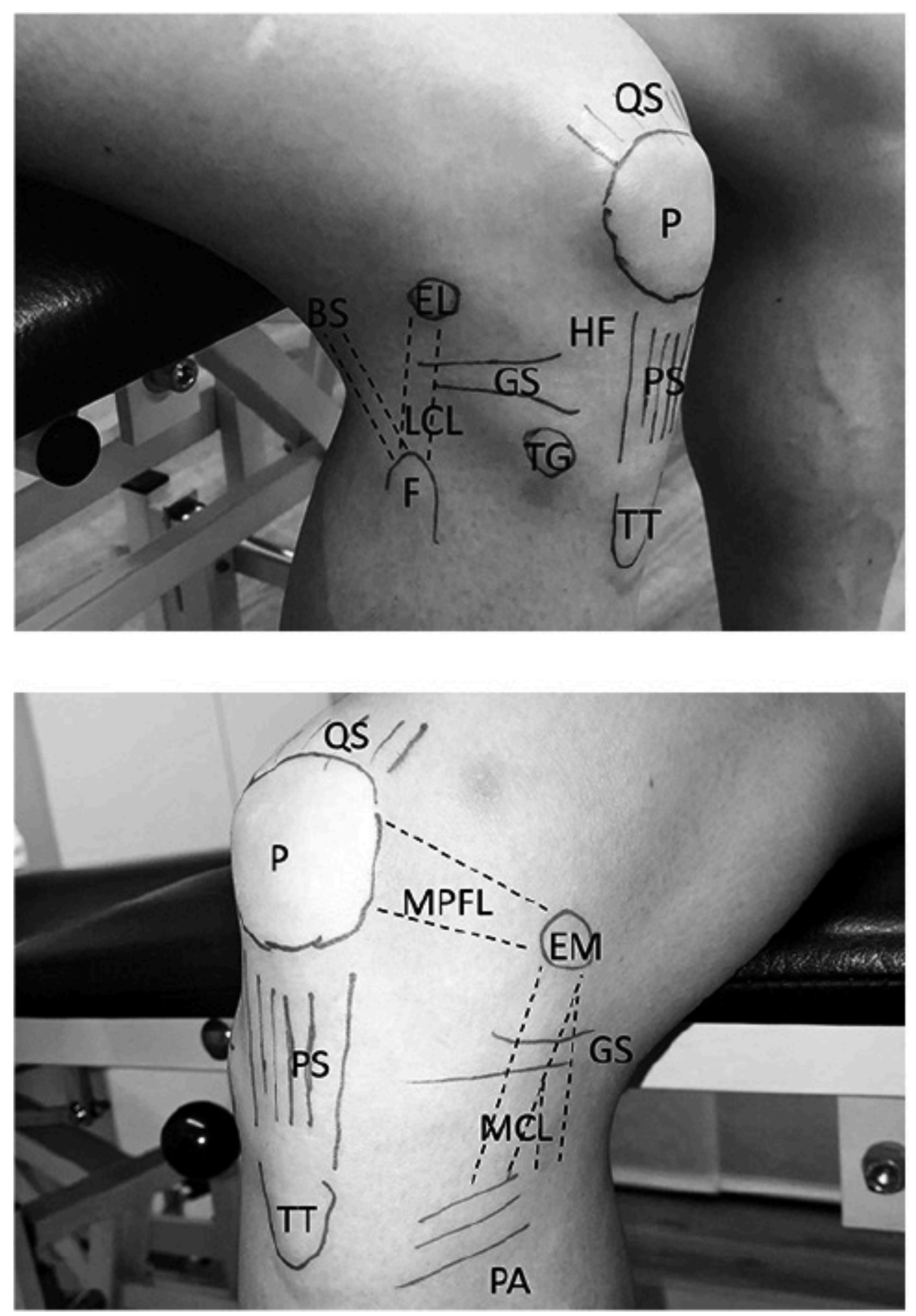

Abb. 4a und b: Landmarken am Kniegelenk von anterolateral (a) und anteromedial (b). QS, Quadricepssehne; P, Patella; PS, Patellarsehne; TT, Tuberositas Tibiae; GS, Gelenkspalt; TG Tuberculum Gerdy (Insertion des Tractus iliotibialis); HF, Hoffa Fettkörper; EL, Epicondylus lateralis; F, Fibulaköpfchen; LCL, Ligamentum collaterale laterale; BS, Bicepssehne; EM, Epicondylus medialis; PA, Pes anserinus; MCL, Ligamentum collaterale mediale; MPFL, Ligamentum patellofemorale mediale. 

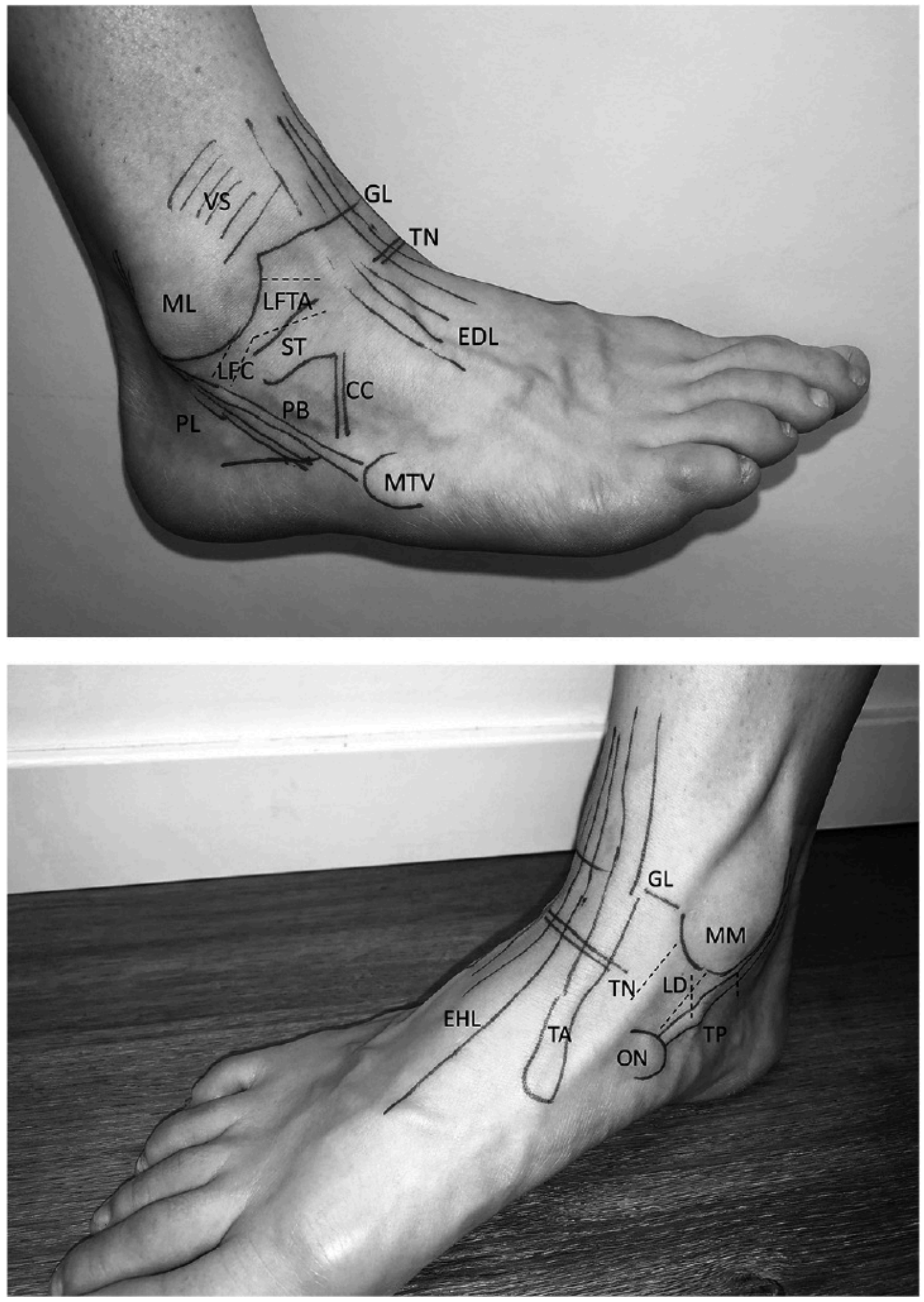


\begin{abstract}
Abb. 5a und b: Landmarken am Rückfuss von anterolateral (a) und anteromedial (b). ML, Malleolus lateralis; GL, Gelenkslinie OSG; TN, Talonavikular-Gelenk; CC, Calcaneo-cuboidal-Gelenk; ST, Sinus Tarsi (Zugang zum USG); VS, Vordere Syndesmose; EDL, Extensor digitorum longus; MTV, Metatarsal V Basis; PB, Peroneus brevis-Sehne; PL, Peroneus longus-Sehne; LFTA, Ligamentum fibulotalare anterius; LFC, Ligamentum fibulocalcaneare; MM, Malleolus medialis, ON, Os naviculare; TP, Tibialis Posterior-Sehne; EHL, Extensor hallucis longus-Sehne; TA, Tibialis anteriorSehne; LD, Ligamentum deltoideum (oberflächliche und tiefe Anteile).
\end{abstract}

\title{
Angiologische Untersuchung
}

Die arteriellen Pulse können an folgenden Lokalitäten getastet werden. A. femoris communis in der Inguina; A. poplitea in der Poplitea leicht lateral der Mitte, A. dorsalis pedis auf dem Os naviculare zwischen Flexor hallucis longus Sehne und Flexor digitorum longus Sehne, A. tibialis im Eingang zum Tarsaltunnel retromalleolär medial. Venöse Veränderungen zeigen sich typischerweise als Ödem um die Knöchel (Abdruck der Socken) oder im Sinne chronischer dermatologischer Veränderungen (Purpura jaune d'ocre oder Atrophie blanche) und im Sinne einer Varikosis (Stammvarikosis V. saphena magna oder parva; Besenreiser).

\section{Untersuchung der Hüfte}

Hüftbeschwerden können sich entweder in die Leiste, nach gluteal oder nach lateral projizieren. Intraartikuläre, coxogene Schmerzen strahlen vornehmlich in die Leiste. Im Umkehrschluss ist jedoch die Differentialdiagnose des Leistenschmerzes sehr breit. Hierbei sei deshalb auf die entsprechende Literatur verwiesen. Bei glutealen Beschwerden muss neben muskulären Ursachen (z.B. Piriformis-Syndrom, Myelogelosen) insbesondere auch an die lumbale Wirbelsäule und das Ilio-sakral-Gelenk gedacht werden. Laterale Beschwerden beziehen oft die Trochanter maior Region mit ein (z.B. Bursitis trochanterica, Coxa saltans externa, Gluteaus medius-Tendinopathie).

Auf folgende spezifisches Tests für das Hüftgelenk möchte hiermit hingewiesen werden: Vorderer Impingementtest (Abb. 6). In Innenrotation und Adduktion wird bei unterschiedlichen Flexionswinkeln (von $120^{\circ}$ bis $30^{\circ}$ Flexion) mit einem aktiven Anschlag versucht, den bekannten Schmerz $\mathrm{zu}$ reproduzieren. Typischerweise ist der Test bei ca $90^{\circ}$ am symptomatischten. Cave: Ein Hüftimpingement kann nur bei reduzierter Innenrotation vorliegen. Bei normaler oder vermehrter Innenrotation muss an eine andere Ursache gedacht werden (isolierte Labrumläsion, Hüftdysplasie usw). Posterior-Rim-Test für ein posteriores Impingement (Abb. 7). Die Umkehr-Bewegung zum vorderen Impingementtest zur Überprüfung des hinteren Pfannenrandes. Hier wird eine Aussenrotationsbewegung in Extension, Abduktion und Aussenrotation der Hüfte durchgeführt.

Faber-Test (Vierer-Position) (Abb. 8). Der Test dient zur Unterscheidung einer coxogenen Ursache (Schmerzlokalisation in der Leiste) versus einer ISG-Problematik (Schmerzursache gluteal-sakral). Dabei wird das Knie, welches in Viererposition liegt, gegen unten gedrückt, um einen Druck auf das Becken auszuüben.

Trendelenburg-Test. Im Einbeinstand muss das Becken stabil gehalten werden. Ein Absinken zur Gegenseite spricht für eine Gluteaus medius Insuffizienz.

Drehmann-Zeichen. Bei aktiver Hüftflexion bewegt sich das flektierte Knie bei positivem Drehmannzeichen in die Hüftabduktion als Zeichen einer coxogenen Pathologie (z.B. Coxarthrose, schweres Impingement). 


\section{Untersuchung des Knies}

Die Untersuchung des Kniegelenks gliedert sich in folgende Abschnitte:

\section{Erguss}

Ergussbildung kann wegweisend sein in der Diagnostik (akuter versus chronischer Erguss; Ergussart: serös, hämatös, putride, kristallogen usw.). Ein massiver Erguss zeigt sich in Form einer Bewegungsunfähigkeit des Gelenks. Der Recessus suprapatellaris ist prall gefüllt. Weniger Erguss lässt sich durch Ausstreichen der Recessus finden. Lokale Ergussbildung kann sich als Zystenbildung (z.B. BakerZyste) oder als Ganglion (z.B. Meniskusganglion) zeigen.

\section{Ligamentäre Stabilität}

Die femoro-tibiale Stabilität des Kniegelenks wird durch die zentrale Stabilität (Vorderes und hinteres Kreuzband) sowie die periphere Stabilität (Innen- und Aussenband, posterolaterale und posteromediale Ecke, Kapsel und verstärkende Anteile) bestimmt. Zentrale und periphere Stabilität können sich gegenseitig beeinflussen (Tab. 3).

Das Innenband wird getestest durch einen Abduktions- oder Valgusstress in Streckung und $30^{\circ}$ Flexion. Isoliert wird das Innenband allerdings nur in $30^{\circ}$ Flexion getestet. Analog verhält es sich mit dem Aussenband, welches mit einem Adduktions- oder Varusstress getestet wird. Bei der Prüfung der Seitenbänder wird dabei die Aufklappbarkeit (+ bis +++) beurteilt sowie die Qualität des Anschlags (weich, hart). Das Dial-Sign besteht aus einer maximalen Aussenrotation der Füsse in Bauchlage und $45^{\circ}$ Knieflexion im Seitenvergleich und ist hinweisend auf eine periphere Kapsel-Band-Instabilität. Das vordere Kreuzband wird bezüglich des Tibiavorschubs in $30^{\circ}$ Knieflexion (Lachman-Test (Abb. 9); Prüfung von Vorschub-Distanz und Anschlag; Klinische Gradierung der Instabilität von + bis +++) und in $90^{\circ}$ Knieflexion (vordere Schublade) gestestet. Das hintere Kreuzband mit der hinteren Schublade, welche aus einem passiven nach posterior Rutschen des Tibiaplateaus besteht. Die Rotationsstabilität als Zeichen einer chronischen vorderen Kreuzbandinstabilität wird mit dem Pivot-shift Test geprüft (Abb. 10). Dabei wird das laterale Tibiaplateau in Knieflexion nach anterior subluxiert. Aufgrund des Zugs des Tractus iliotibialis kommt es in einer Drehbewegung zur Streckung hin zu einer symptomatischen Reposition, was beweisend für eine Rotationsinstabilität (Pivotieren) ist.

\section{Meniskus und Knorpel}

Meniskus und Knorpel haben unter anderem eine dämpfende Funktion im Gelenk. Sie beeinflussen sich gegenseitig sehr stark und sind klinisch nicht einfach voneinander zu differenzieren. Im Unterschied zu den Ligamenten, bei welchen eine Testung aus der Dehnung des Ligaments besteht, wird für die Meniskustestung der Meniskus komprimiert. Es ist deshalb wichtig zu verstehen, dass zum Beispiel ein Valgusstresstest bei medialen Schmerzen (Dehnung) auf eine Innenbandverletzung hinweisen kann, bei einem lateralen Schmerz (Kompression) auf eine Aussenmeniskusverletzung. Kann eine maximale Extension und Flexion schmerzfrei durchgeführt werden, ist eine Meniskusverletzung bereits sehr unwahrscheinlich. Zusätzlich existieren jedoch unzählige weitere Tests, bei denen auf die eine oder andere Art ein Anteil des Meniskus unter Kompression gebracht wird. Einige wichtige seien hier erwähnt (Tab. 4). 
McMurray-Test: Knierotation in maximaler Flexion und Bewegung bis zur $90^{\circ}$ Position. Die Weiterführung des Tests bis zur vollen Streckung wird als Bragard-Test bezeichnet. Bei zusätzlichem Varus- oder Valugsstress wird der Test als Payr-Test bezeichnet.

Steinmann I und II-Test Analog zum Mc-Murray Test wird die Rotation in verschiedenen Knieflexionsstellungen durchgeführt. Zusätzlich wird das Kniegelenk axial komprimiert. Während beim Steinmann I die Knieflexion während jedem Test fixiert bleibt, wird beim Steinman II zusätzlich mit der Extension-Flexion gespielt.

Apley-Grinding Test: Untersuchung des Knies in Bauchlage, was sich insbesondere bei schweren Beinen anbietet. Durchführen einer Kompression in Abduktion-Innenrotation oder Adduktion-Aussenrotation. Thessaly-Test: Untersuchung des Knies im Stehen. In $5^{\circ}$ und $20^{\circ}$ Knieflexion wird unter Belastung auf dem verletzten Knie rotiert.

\begin{tabular}{|c|c|c|c|}
\hline Ligament & Primäre Funktion & Sekundäre Funktion & Synergistisch zu \\
\hline Vorderes Kreuzband & $\begin{array}{l}\text { Begrenzt anterolaterale Ver- } \\
\text { schieblichkeit der Tibia im } \\
\text { Vergleich zum Femur }\end{array}$ & $\begin{array}{l}\text { Begrenzt Valgusstress in } \\
\text { Streckung und Varusstress in } \\
\text { Flexion }\end{array}$ & $\begin{array}{l}\text { Posterolateraler Ecke, } \\
\text { Innenband }\end{array}$ \\
\hline Hinteres Kreuzband & $\begin{array}{l}\text { Begrenzt posteriore Tibiaver- } \\
\text { schieblichkeit, insbesondere in } \\
\text { Knieflexion }\end{array}$ & Begrenzt Varusstress & Aussenband \\
\hline Innenband & Begrenzt den Valgusstress & $\begin{array}{l}\text { Unterstützt das VKB in der } \\
\text { Rotationskontrolle }\end{array}$ & Vorderem Kreuzband \\
\hline Aussenband & $\begin{array}{l}\text { Begrenzt den Varusstress } \\
\text { insbesondere im mittleren } \\
\text { Flexionsbereich }\end{array}$ & $\begin{array}{l}\text { Begrenzt die Knierotation vor } \\
\text { allem Streckungsnahe }\end{array}$ & Posterolateraler Ecke \\
\hline Posterolaterale Ecke & $\begin{array}{l}\text { Kontrolliert die Rotation der } \\
\text { Tibia im Verhältnis zum Femur }\end{array}$ & $\begin{array}{l}\text { Begrenzt Varusstress und } \\
\text { posteriore Tibiaverschieblichkeit }\end{array}$ & Hinterem Kreuzband \\
\hline
\end{tabular}




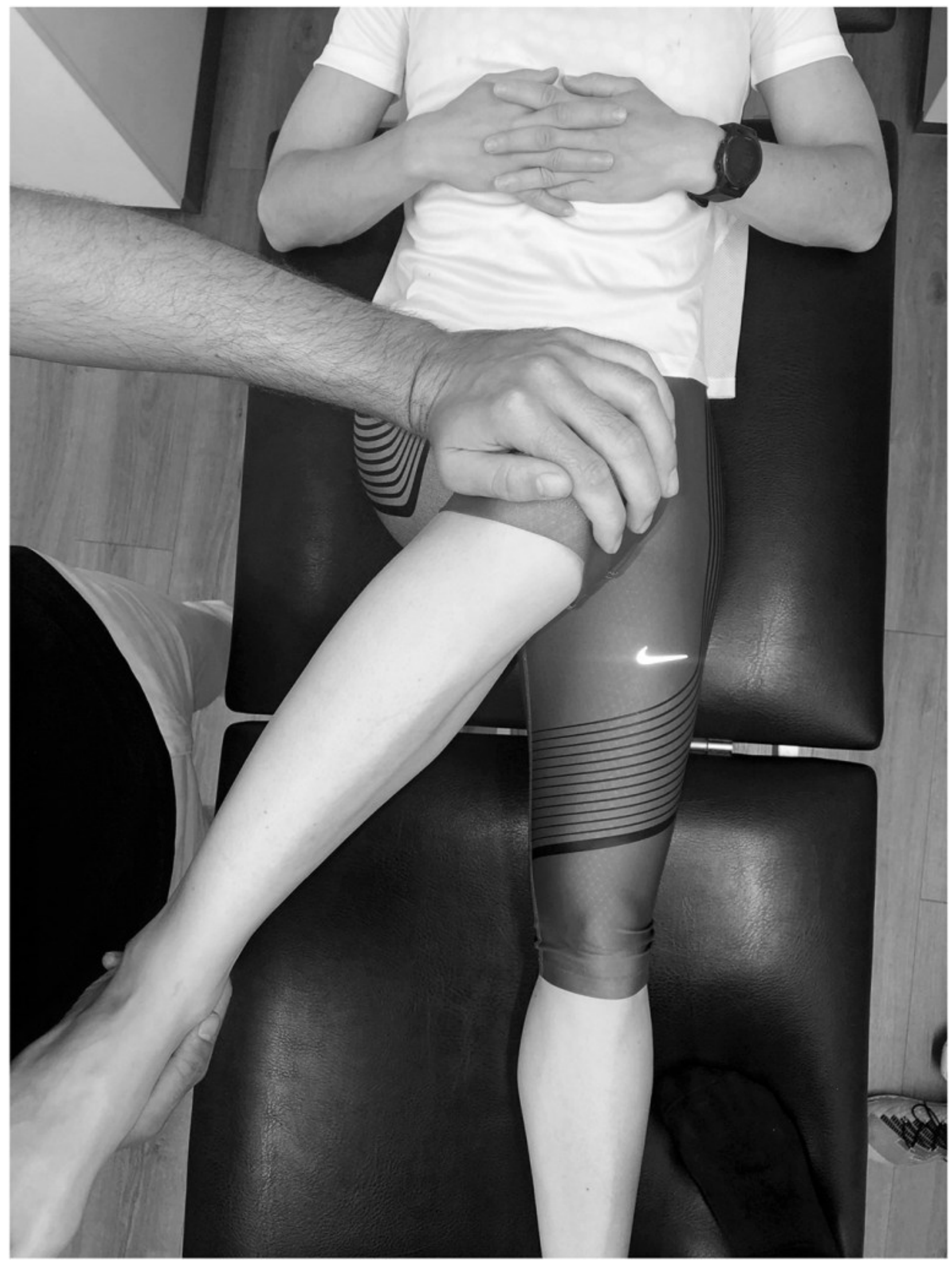


Abb. 6: Vorderer Impingement-Test. In Rückenlage wird in Innenrotation und Adduktion in verschiedenen Flexionsgraden (von $120^{\circ}$ bis $30^{\circ}$ Flexion) ein Einklemmen durch forcierte Adduktion ausgelöst und ist positiv, wenn damit der bekannte Schmerz ausgelöst werden kann. 


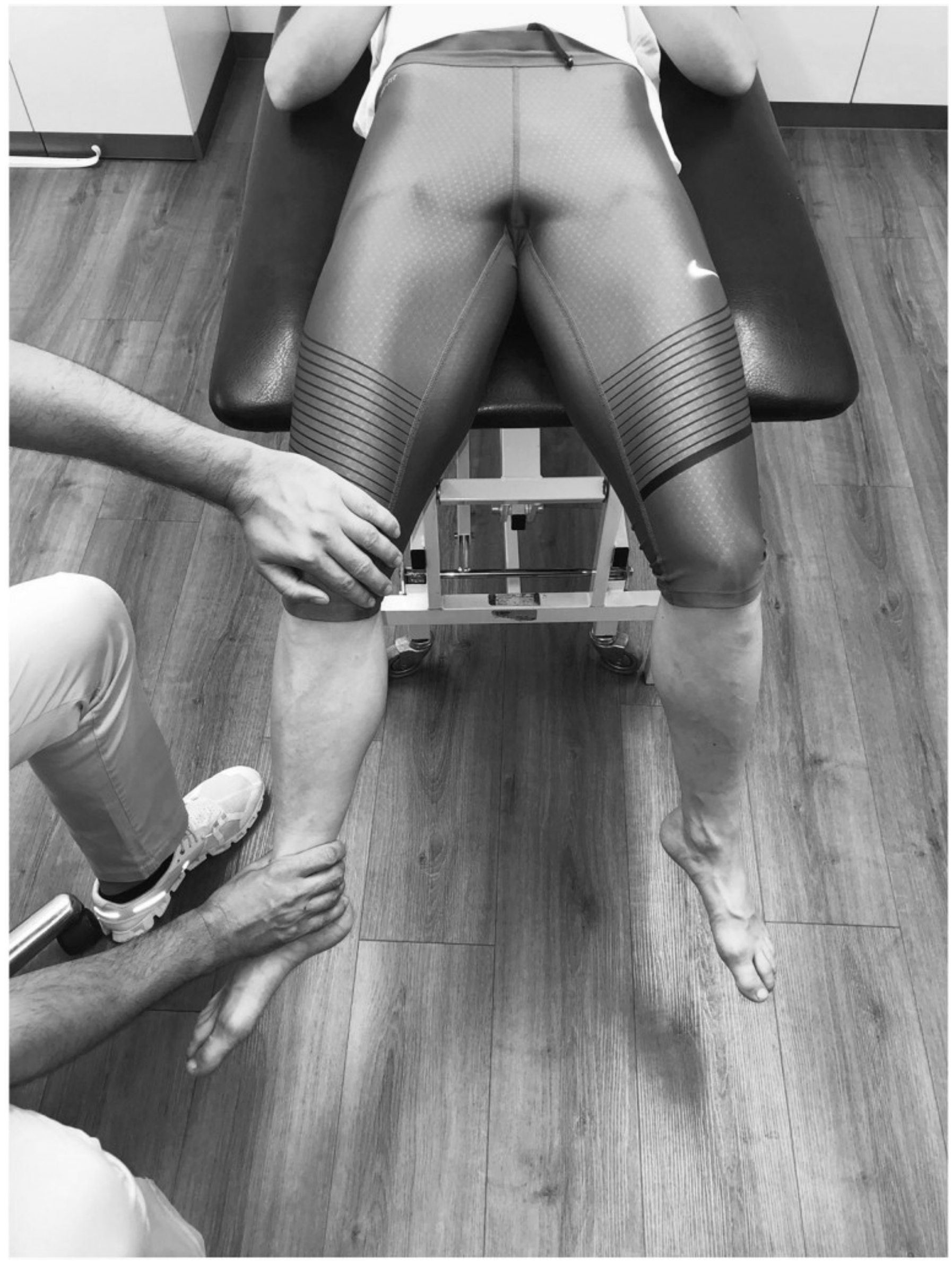


Abb. 7: Posterior-Rim-Test. Test für den hinteren Pfannenrand in Extension Abduktion und forcierter Aussenrotation (spiegelbildlich zum vorderen Impingementtest). 


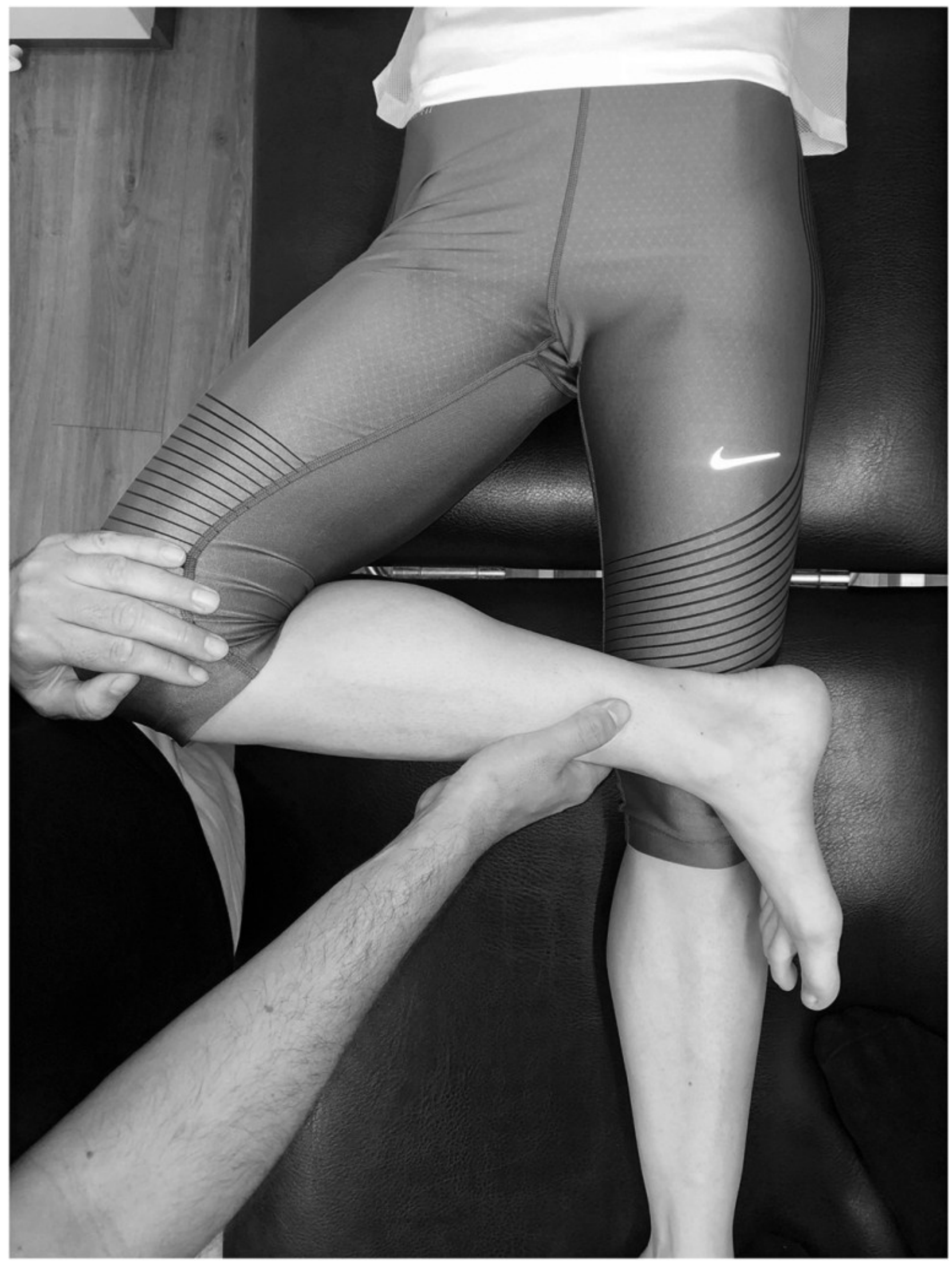


Abb. 8: Vierer-Test/Faber-Test. Durch Druck des Knies nach unten wird ein Druck auf den hinteren Beckenring, namentlich das ISG ausgelöst. 


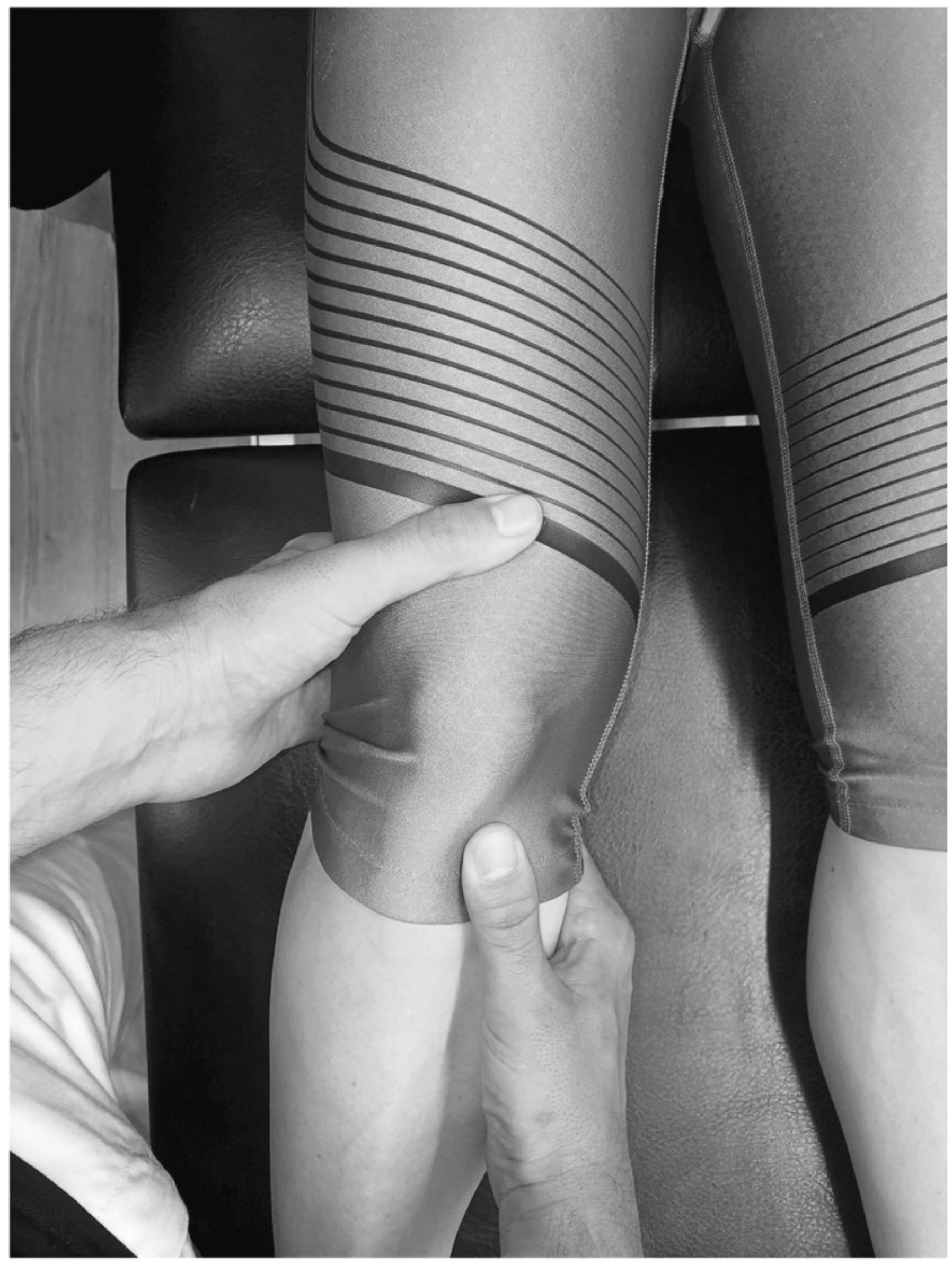


Abb. 9: Lachman-Test. In 20-30 Knieflexion wird der Tibiavorschub geprüft zur Überprüfung des vorderen Kreuzbandes. 


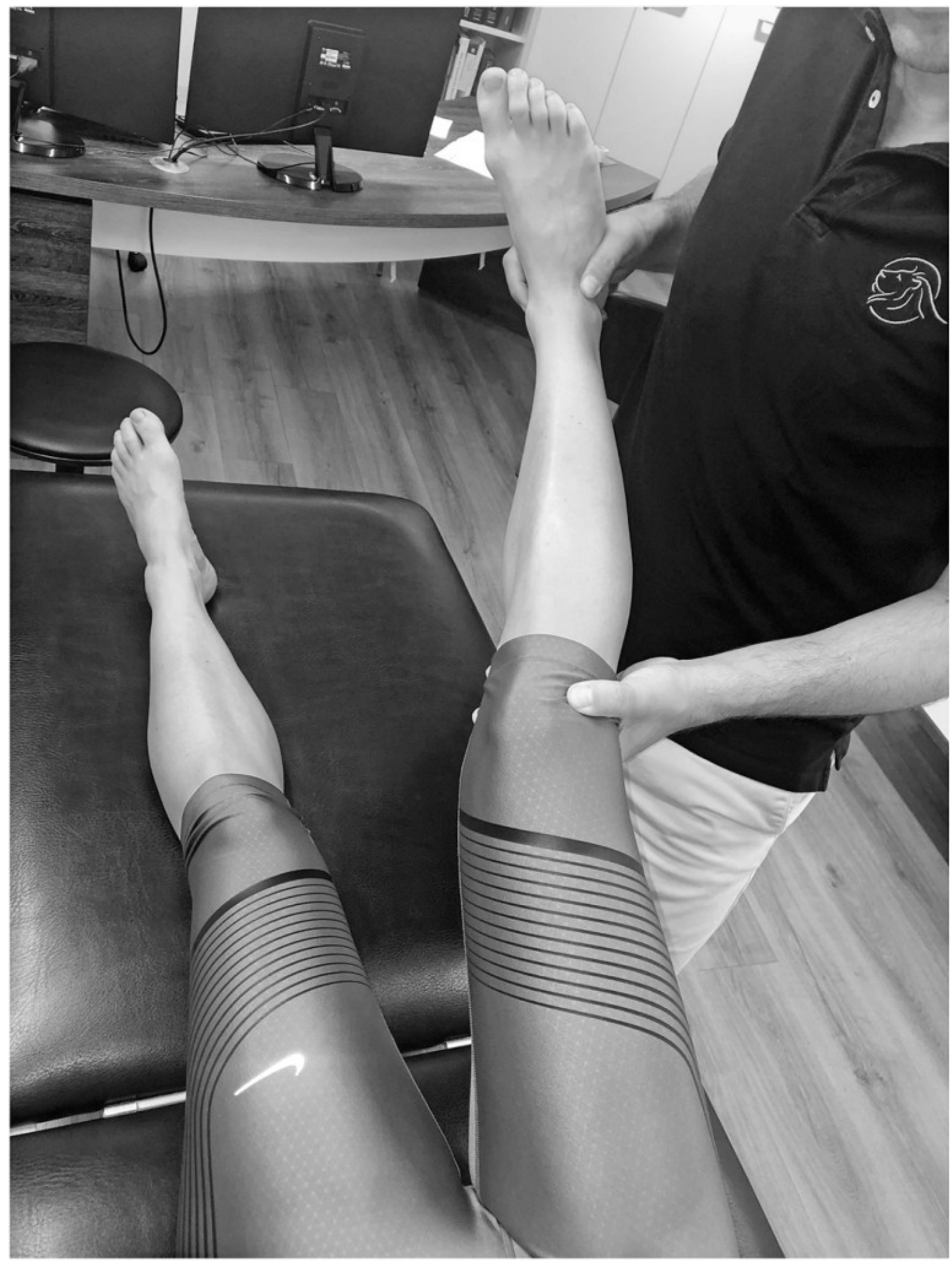


Abb. 10: Pivot-Shift-Test. Zur Überprüfung der Rotationsstabilität des Kniegelenks wird bei leicht flektiertem, adduziertem Knie das Tibiaplateau versucht, nach anterolateral zu subluxieren. In der Bewegung hin zur Extension kommt es zu einer symptomatischen Reposition bei Rotationsinstabilität.

\section{Patello-femoral Gelenk}

Das Patello-femoral Gelenk verdient besondere Aufmerksamkeit, ist es doch oft isoliert vom femorotibialen Kniegelenk zu untersuchen. In Extension kann die Stabilität der Patella geprüft werden, respektive die medio-laterale Verschieblichkeit. Diese soll maximal zwei Quadranten der Patellabreite betragen, zudem soll sie mind. $45^{\circ}$ lateral evertiert werden können. Das Zohlen-Zeichen ist klinisch wenig zielführend und kann immer schmerzhaft ausgestaltet werden. Zusätzlich sind Druckdolenzen rund um die Patella zu prüfen (Insertionstendinopathie/-tendinitis der Quadricepssehne proximal und der Patellarsehne distal; Plicasyndrom medial inferior, MPFL Insertion medial und laterales Retinaculum lateral). Retropatellare Knorpelschäden zeigen oft ein Krepitieren bei Bewegung unter Druck. Bei schlanken Patienten kann auch die Trochlea palpiert werden. Bei Verdacht auf eine Patello-femorale Problematik ist jedoch oft eine dreidimensionale Bildgebung (insb. MRI) unverzichtbar zur Erfassung aller strukturell-mechanischer Begleitfaktoren (Trochleadysplasie, Patella alta/baja, TTTG-Winkel usw.). Ein positives Apprehension-Zeichen (analog der Schulter) kann hinweisend für eine stattgehabte Patellaluxation sein.

\section{Untersuchung von Sprunggelenk und Fuss}

Neben den bereits weiter oben erwähnten Untersuchungen des Sprunggelenks und Fusses (Inspektion und Palpation) interessiert uns insbesondere die Stabilität des Rückfusses.

Anterior Drawer Sign / Talusvorschub (Abb. 11): Untersuchung (z. B. im Sitzen) bei hängendem, im Knie flektierten Bein zur Prüfung der Stabilität nach anterior (Lig. Fibulotalare anterius und anteromediale, insb. oberflächliche Anteile des Lig. Deltoideum). Einteilung der Instabilität in + bis +++.

Lateral Talar Tilt Sign/Laterale Aufklappbarkeit (Abb. 12). Untersuchung bei hängendem, im Knie flektierten Bein (z. B. im Sitzen) zur Prüfung der Stabilität lateral. Einteilung der Instabilität in + bis +++. Hierbei wird insbesondere das Lig. Fibulocalcaneare beurteilt.

Medial Talar Tilt Sign/Mediale Aufklappbarkeit (Abb. 13). Untersuchung bei hängendem, im Knie flektierten Bein (z. B. im Sitzen) zur Prüfung der Stabilität medial. Einteilung der Instabilität in + bis +++. Hierbei werden insbesondere die posterioren Anteile des Lig. Deltoideums beurteilt. Bei subtilen chronischen medialen Instabilitäten ist insbesondere die Aussenrotation in 10-20 Plantarflexion beim hängenden OSG vermehrt hinweisend auf eine Insuffizienz der anteromedialen Bandanteile (Tibio-SpringAnteile und Tibio-navicularen Anteile des Lig. Deltoideums).

Zur Untersuchung der Syndesmose werden folgende Tests hinzugezogen: (I) Verschieblichkeit des Malleolus lateralis in antero-posteriore Richtung, (II) Druckdolenz auf der vorderen Syndesmose, (III) positiver Syndesmosen Squeeze-Test (Zusammendrücken von Fibula und Tibia auf Unterschenkelmitte löst distal einen Schmerz aus), und (IV) schmerzhafte Aussenrotation in Dorsalextension im OSG.

Zur aktiven Krafttestung gehört insbesondere die Pronationskraft (Peroneus brevis- und Longus-Sehnen) sowie die Untersuchung der Supination (Tibialis Posterior Sehne). Hierzu muss der Rückfuss im Zehenspitzenstand eine Varisation der Ferse zeigen. Die Aktivität des Tibialis Posterior Muskels führt bei 
intakter Sehne im Zehenspitzenstand zu einer Varisation des Rückfusses (zu sehen als Inversion der Ferse von hinten).

Die Achillessehne unterscheidet anatomisch drei Regionen. Proximal der Muskulo-tendinöse Übergang, der mid- portion-Bereich (klassische Achillessehnen-Tendinopathie) und der Insertionsbereich distal. Die Untersuchung der Achillessehne erfolgt zusätzlich in Bauchlage. Zur Längenbestimmung wird der Fuss über den Liegenrand hängen gelassen (Hanging Foot Sign als Zeichen einer verlängerten Sehne; Abb. 14). Der Thompson-Test untersucht die Kraftüberleitung von Wadenmuskulatur auf den Fuss.

\section{Untersuchung der Muskeln}

Für die Diagnostik von Muskelverletzungen und -beschwerden ist nach wie vor die klinische Untersuchung das wichtigste Werkzeug. Zwar können strukturelle Muskelschäden im MRI oder im Ultraschall dargestellt werden, die Mehrzahl der Muskelverletzungen sind jedoch funktioneller Genese und zeigen daher in der Regel in der Bildgebung keine pathologischen Befunde. Zugleich stellt die Muskulatur den relevanten Motor jeder Bewegung dar und ist für den Sportler/die Sportlerin das zentrale Gewebe für die Leistungsfähigkeit. Es bedarf daher einem speziellen Augenmerk. Erfreulicherweise ist der Muskel das Gewebe was für Trainierbarkeit, aber auch Verletzungsprävention am besten zugänglich ist. Die Muskulatur kann bezüglich folgender Qualitäten untersucht werden:

- Muskeltrophik

- Muskellänge (Abb. 15a-e)

- Kraft

- Muskeldehnungsschmerz

- Aktivierungsschmerz in Endposition (isometrisch, konzentrisch, exzentrisch)

- Aktivierungsschmerz in Neutralposition (isometrisch, konzentrisch, exzentrisch)

- Triggerpunkte/Myelogelosen

Neben der genauen Palpation der Muskulatur kann jede Muskelgruppe isoliert geprüft werden (nach Fromm et al.). Die Muskellänge wird dabei in Winkelgrad $\left(^{\circ}\right)$ des jeweiligen Gelenks angegeben. Damit wird die Grössenordnung unabhängig von der Körpergrösse (Tab. 5).

\begin{tabular}{|c|c|c|c|c|c|c|}
\hline Test & Sensitivität & Spezifität & PPV & NPV & LR+ & LR- \\
\hline $\begin{array}{l}\text { Druckschmerz über } \\
\text { dem Gelenkspalt }\end{array}$ & $\begin{array}{l}64 \% \\
{[62-66 \%]}\end{array}$ & $\begin{array}{l}61 \% \\
(59-63 \%)\end{array}$ & $\begin{array}{l}62 \% \\
160-64 \%)\end{array}$ & $\begin{array}{l}63 \% \\
{[61-65 \%]}\end{array}$ & $\begin{array}{l}1,6 \\
(1,5 ; 1,8]\end{array}$ & $\begin{array}{l}0,59 \\
{[0,54 ; 0,65]}\end{array}$ \\
\hline McMurray & $\begin{array}{l}51 \% \\
{[48-53 \%]}\end{array}$ & $\begin{array}{l}78 \% \\
(77-80 \%)\end{array}$ & $\begin{array}{l}70 \% \\
{[68-72 \%]}\end{array}$ & $\begin{array}{l}61 \% \\
{[59-63 \%]}\end{array}$ & $\begin{array}{l}2,3 \\
(2,1 ; 2,6]\end{array}$ & $\begin{array}{l}0,63 \\
(0,59 ; 0,68)\end{array}$ \\
\hline Apley-Grinding & $\begin{array}{l}38 \% \\
{[36-41 \%]}\end{array}$ & $\begin{array}{l}84 \% \\
(82-86 \%)\end{array}$ & $\begin{array}{l}71 \% \\
\mid 68-74 \%)\end{array}$ & $\begin{array}{l}58 \% \\
{[55-60 \%]}\end{array}$ & $\begin{array}{l}2,4 \\
(2,0 ; 3,0)\end{array}$ & $\begin{array}{l}0,73 \\
{[0,68 ; 0,78)}\end{array}$ \\
\hline Thessaly & $\begin{array}{l}91 \% \\
{[88-94 \%]}\end{array}$ & $\begin{array}{l}97 \% \\
(95-99 \%)\end{array}$ & $\begin{array}{l}97 \% \\
(95-99 \%)\end{array}$ & $\begin{array}{l}91 \% \\
{[89-94 \%]}\end{array}$ & $\begin{array}{l}31,1 \\
{[19,4 ; 72,4]}\end{array}$ & $\begin{array}{l}0,09 \\
(0,06 ; 0,12)\end{array}$ \\
\hline
\end{tabular}




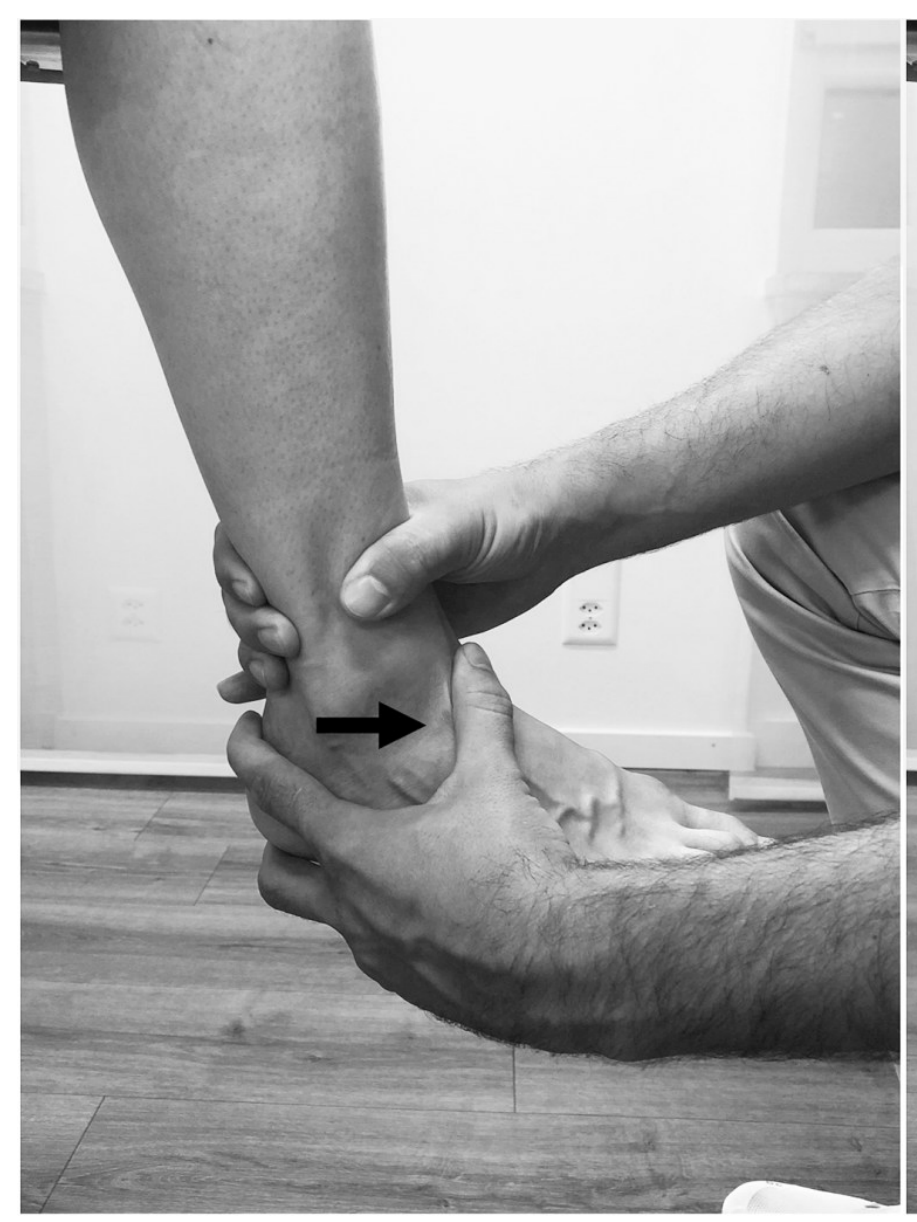

Abb. 11: Anterior-Drawer-Test/Talusvorschub: Überprüfung der OSG-Stabilität in antero-posteriorer Richtung. Durchführung des Tests in sitzender Position mit $90^{\circ}$ Knieflexion.

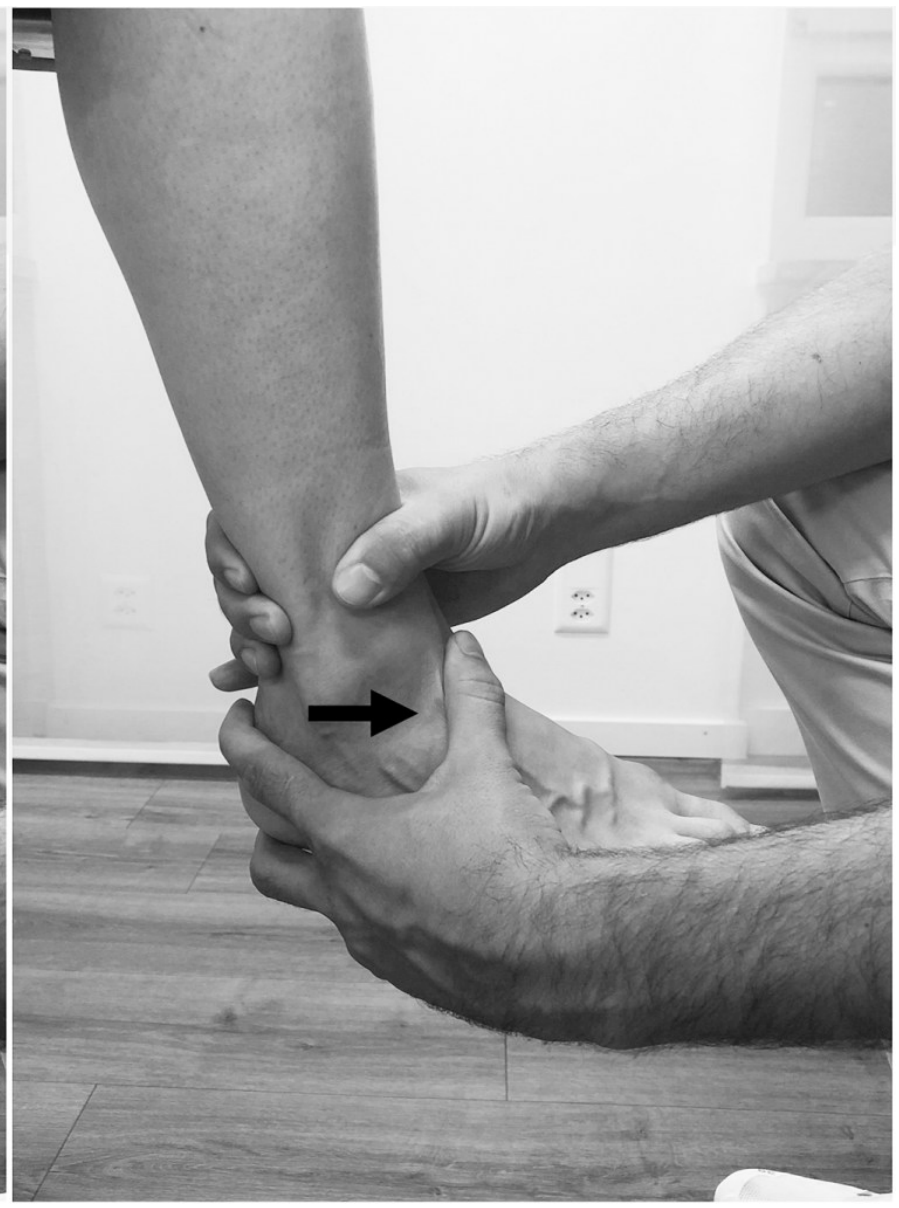

Abb. 12: Lateral-talar-tilt-Test/Laterale Aufklappbarkeit: Überprüfung des lateralen Bandapparates. Durchführung des Tests in sitzender Position mit $90^{\circ}$ Knieflexion. 


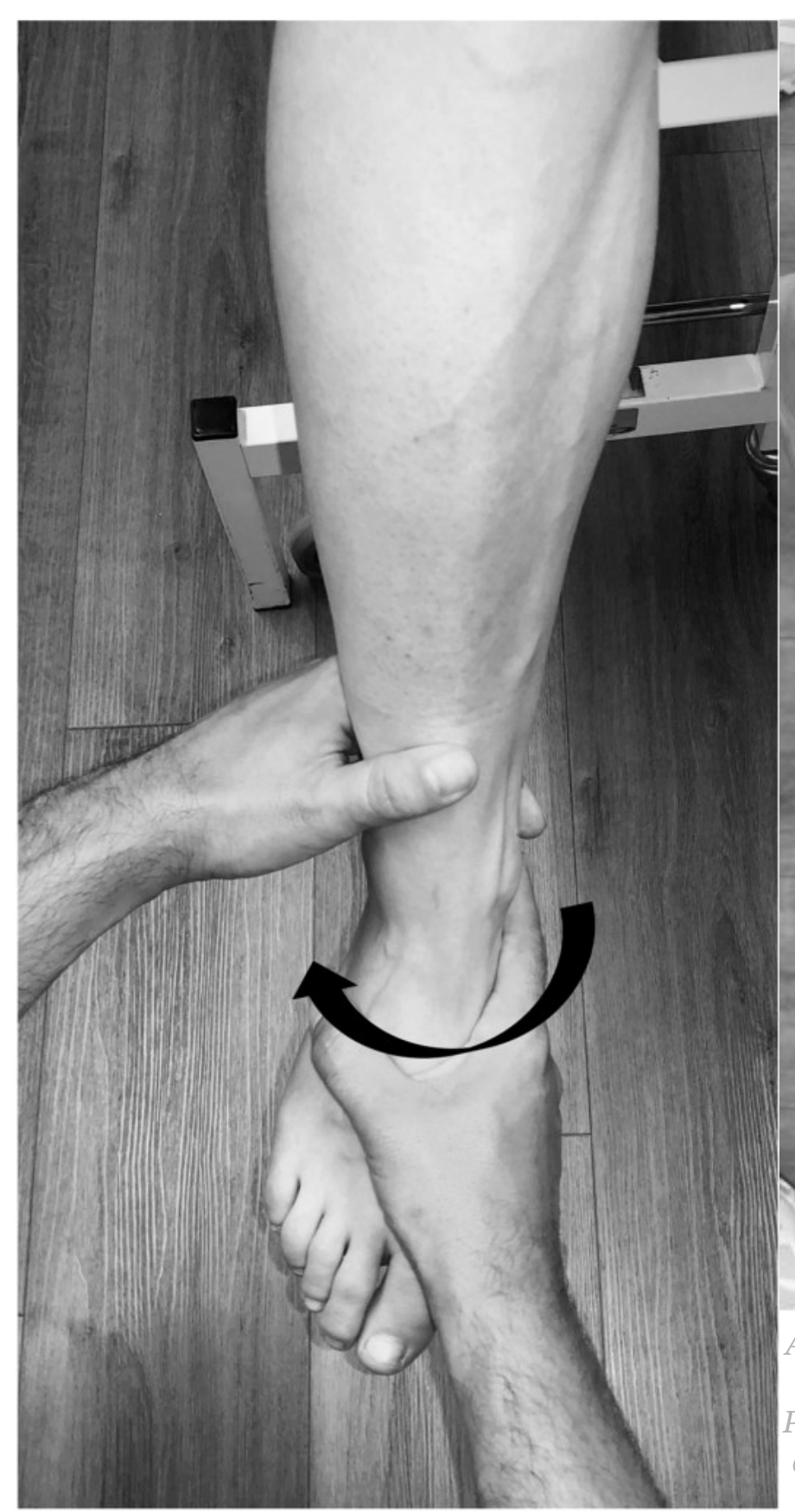

Abb. 13: Medial-talar-tilt-Test/Mediale

Aufklappbarkeit: Überprüfung des medialen Bandapparates. Bei Verdacht auf chronisch mediale Instabilität ist auch die Rotation bei leicht flektiertem OSG im Seitenvergleich zu überprüfen.

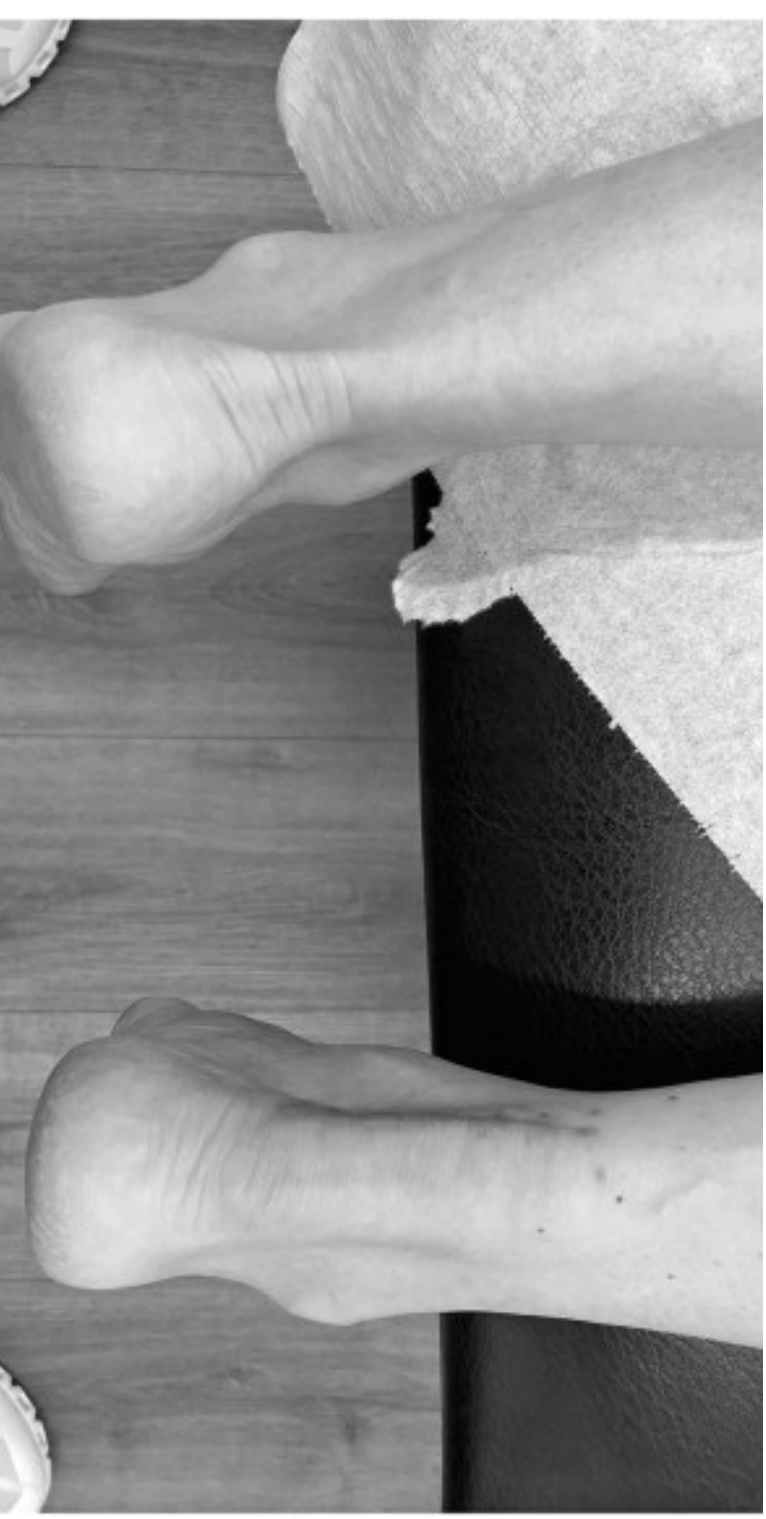

Abb. 14: Hanging Foot Sign. Eine intakte Achillessehne führt aufgrund ihrer Vorspannung zu einer leichten Plantarflexion des Fusses in Bauchlage. Zum Vergleich die postoperativ zu lange Sehne auf der rechten Seite, wo der Fuss ohne Vorspannung nach unten hängt. 


\begin{tabular}{|c|c|c|c|}
\hline Muskel /-gruppe & Position & Winkel & Abbildung \\
\hline Hüftbeuger & $\begin{array}{l}\text { Rückenlage, ein Knie auf dem } \\
\text { Brustkorb, anderes Bein über die } \\
\text { Liege überhängend }\end{array}$ & $\begin{array}{l}\text { Winkel zwischen Liege und über- } \\
\text { hängender Oberschenkellängs- } \\
\text { achse }\end{array}$ & $15 a$ \\
\hline Rectus femoris & Bauchlage, Fuss Richtung Gesäss & $\begin{array}{l}\text { Winkel zwischen Ober- und Unter- } \\
\text { schenkellängsachse }\end{array}$ & $15 b$ \\
\hline Hamstrings & $\begin{array}{l}\text { Rückenlage, mit extendiertem Knie, } \\
\text { Bein Richtung Decke }\end{array}$ & $\begin{array}{l}\text { Winkel zwischen Liege und Ober- } \\
\text { schenkellängsachse }\end{array}$ & $15 c$ \\
\hline Adduktoren & $\begin{array}{l}\text { Rückenlage, ein Bein über Liegen- } \\
\text { rand herabhängend (Fixation hori- } \\
\text { zontale SIAS), anderes Bein mit } \\
\text { extendiertem Knie abduziert }\end{array}$ & $\begin{array}{l}\text { Winkel zwischen Liegenlängsachse } \\
\text { und Beinlängsachse }\end{array}$ & $15 d$ \\
\hline Triceps surae & $\begin{array}{l}\text { Schrittstellung mit Ferse auf dem } \\
\text { Boden, Knie gestreckt, Becken so } \\
\text { weit nach vorne wie möglich }\end{array}$ & $\begin{array}{l}\text { Winkel zwischen Boden und Unter- } \\
\text { schenkellängsachse }\end{array}$ & $15 e$ \\
\hline
\end{tabular}




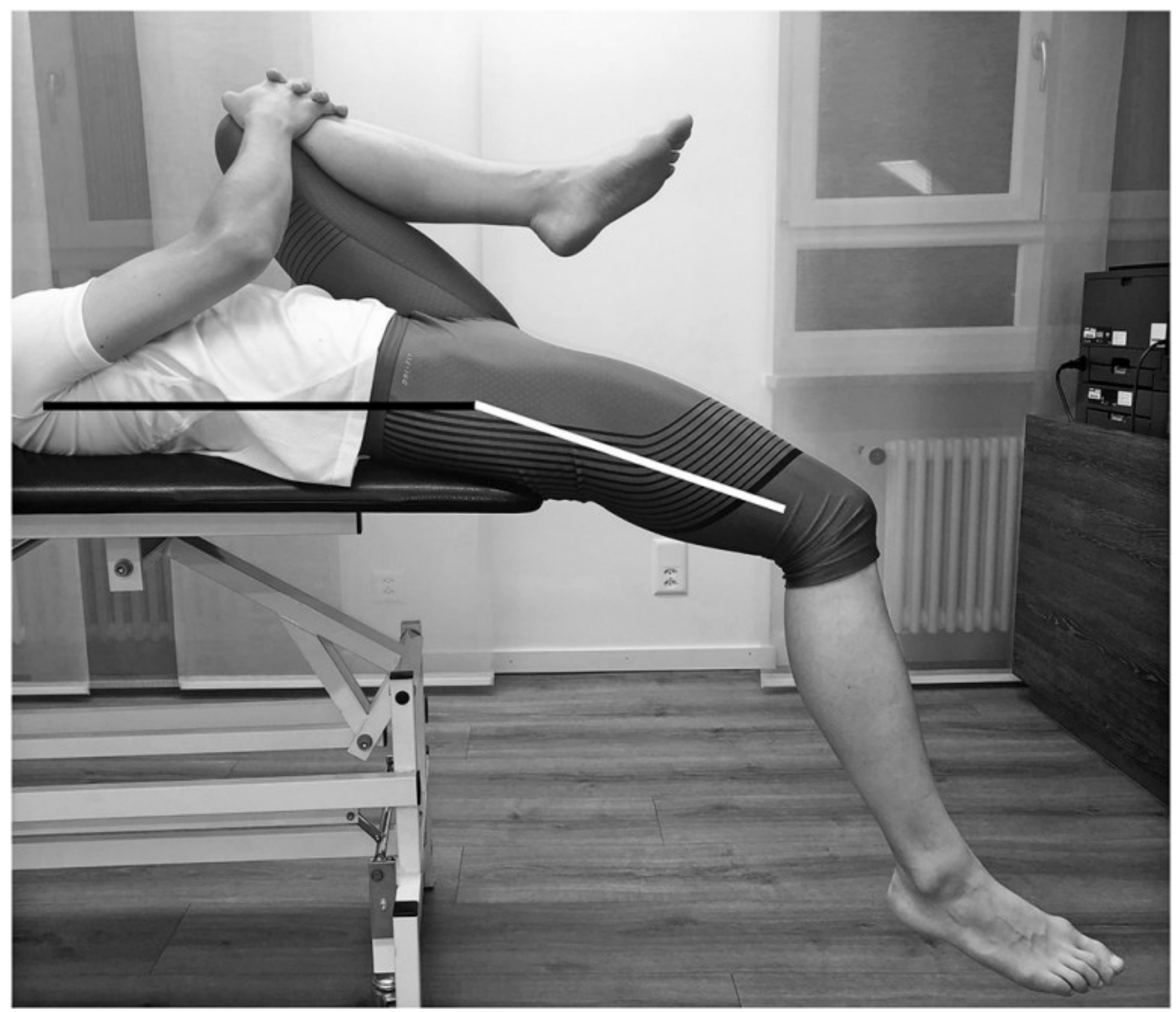




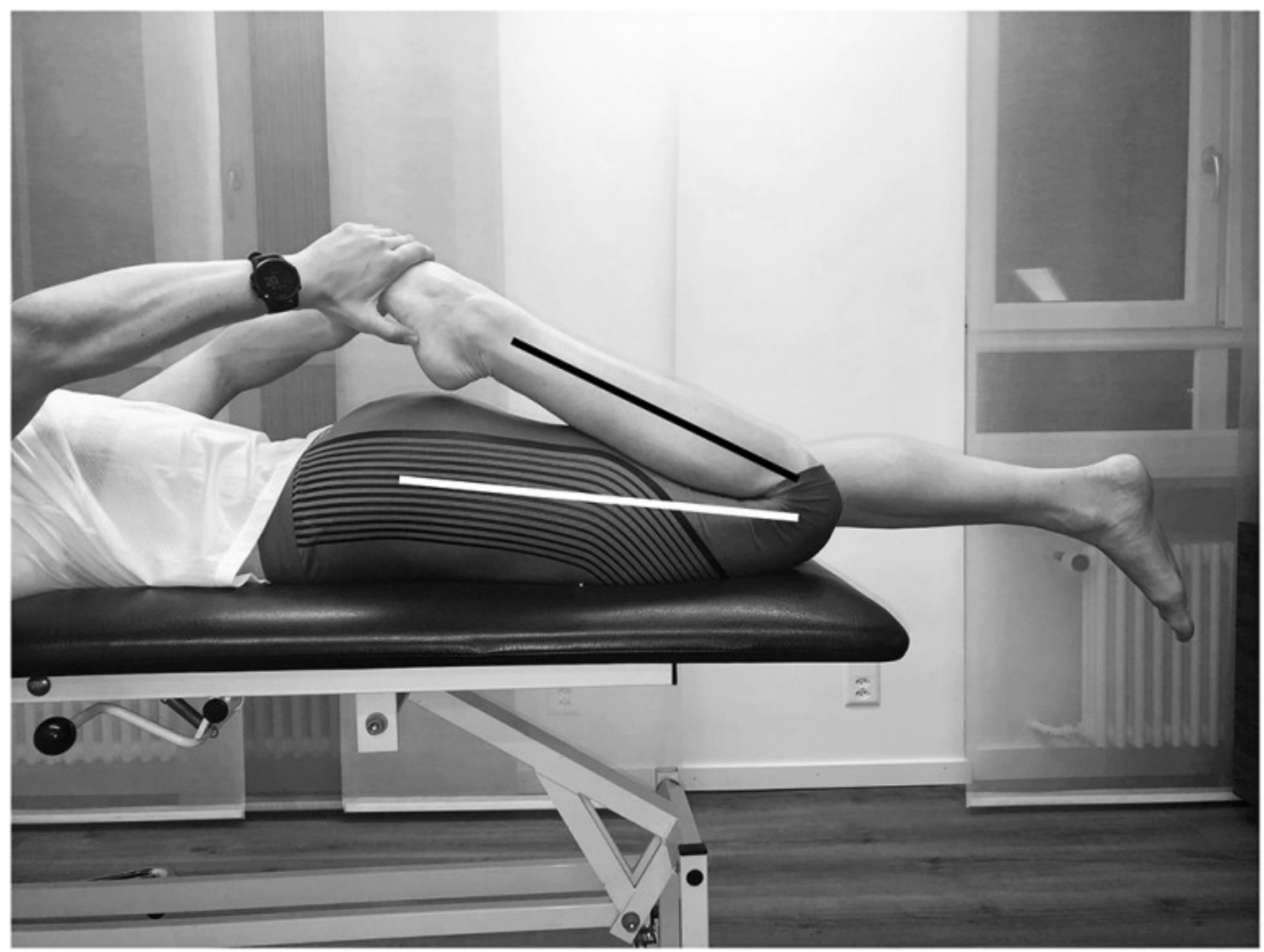




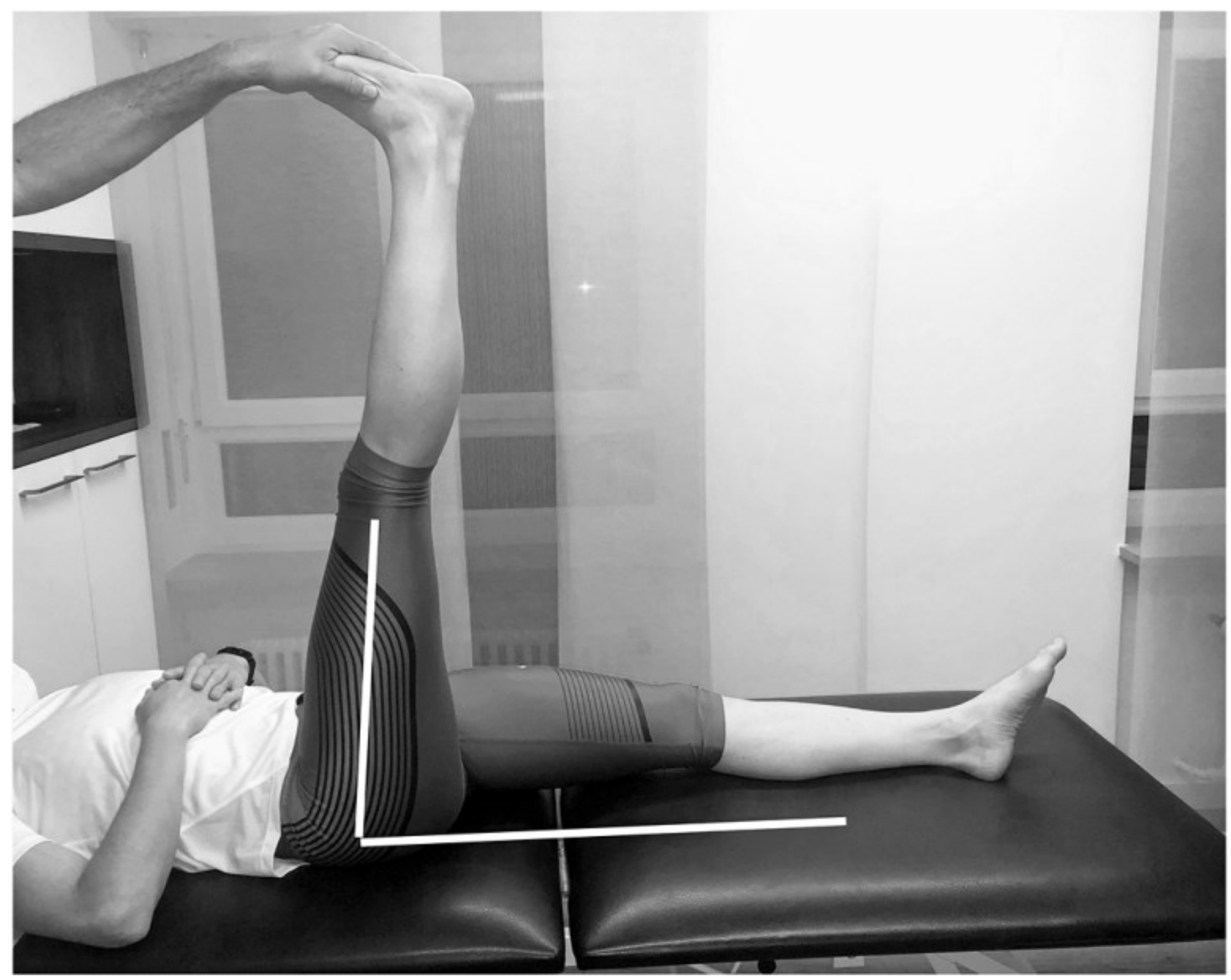




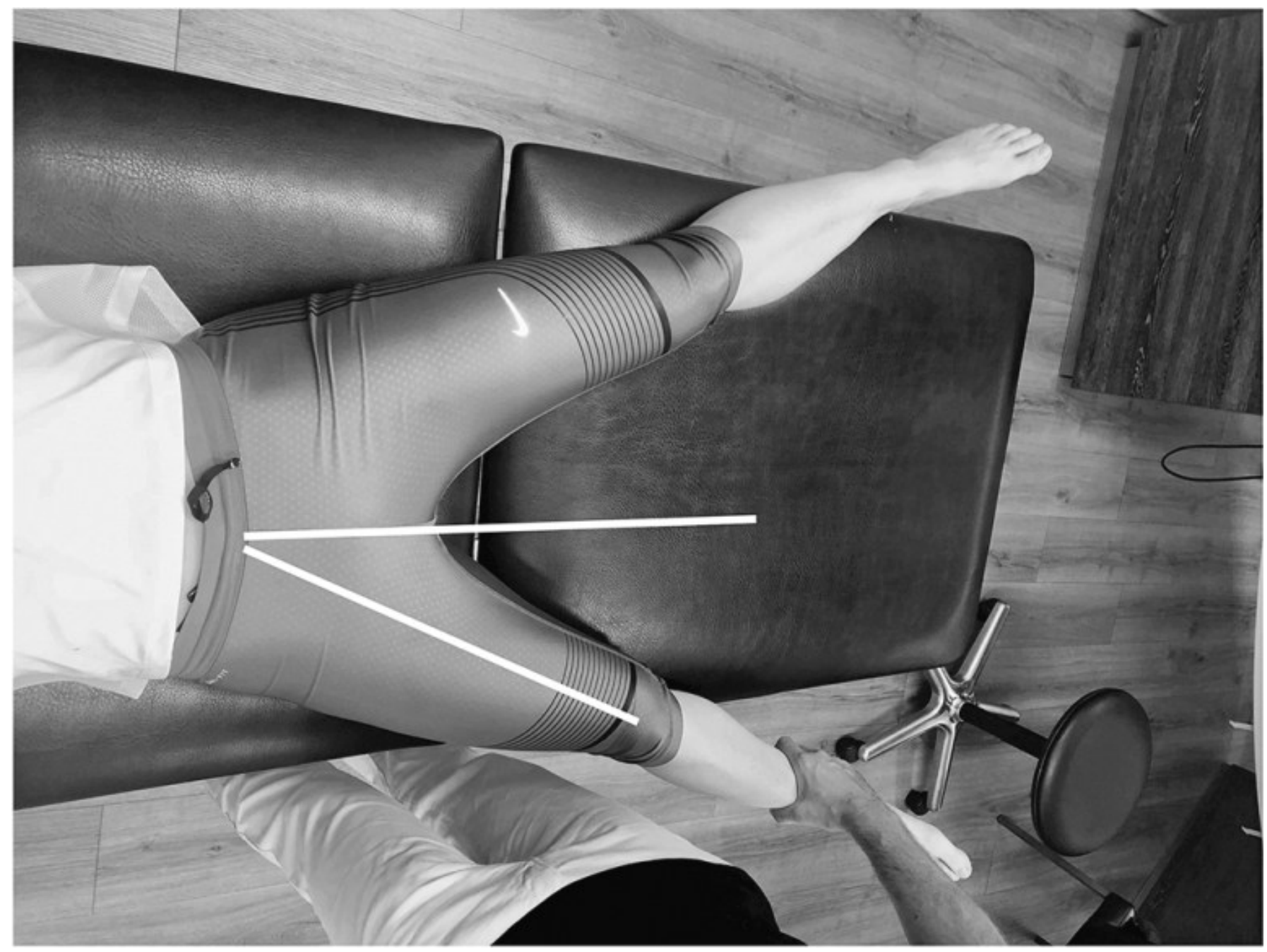




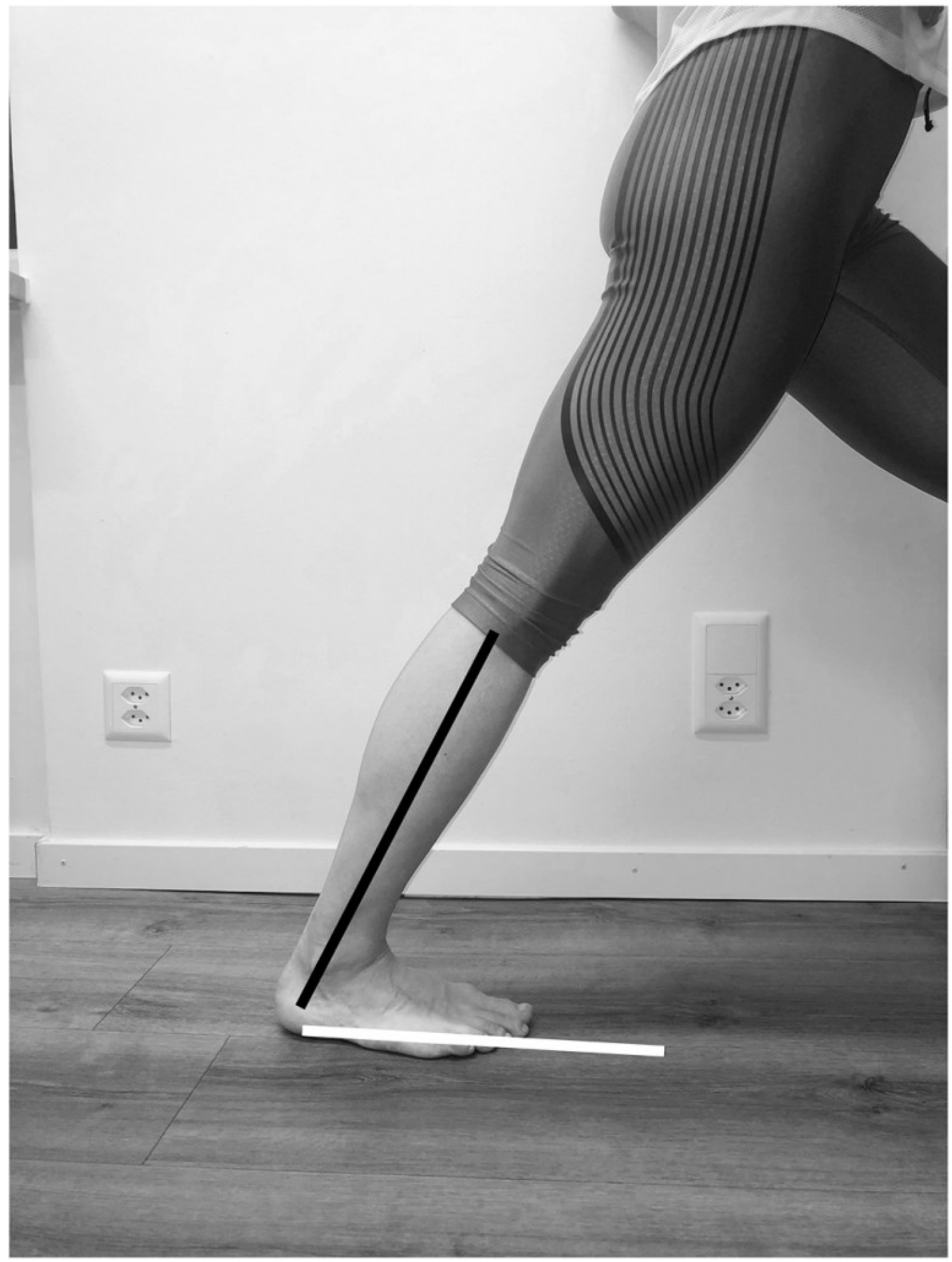


Abb. 15 a-e: Muskellängenmessung. (a) Hüftbeuger: Winkel zwischen Liege und überhängender Oberschenkellängsachse. (b) Rectus femoris: Winkel zwischen Ober- und Unterschenkellängsachse. (c) Hamstrings: Winkel zwischen Liege und Oberschenkellängsachse. (d) Adduktoren: Winkel zwischen Liegelängsachse und Beinlängsachse. (e) Triceps surae: Winkel zwischen Boden und Unterschenkellängsachse.

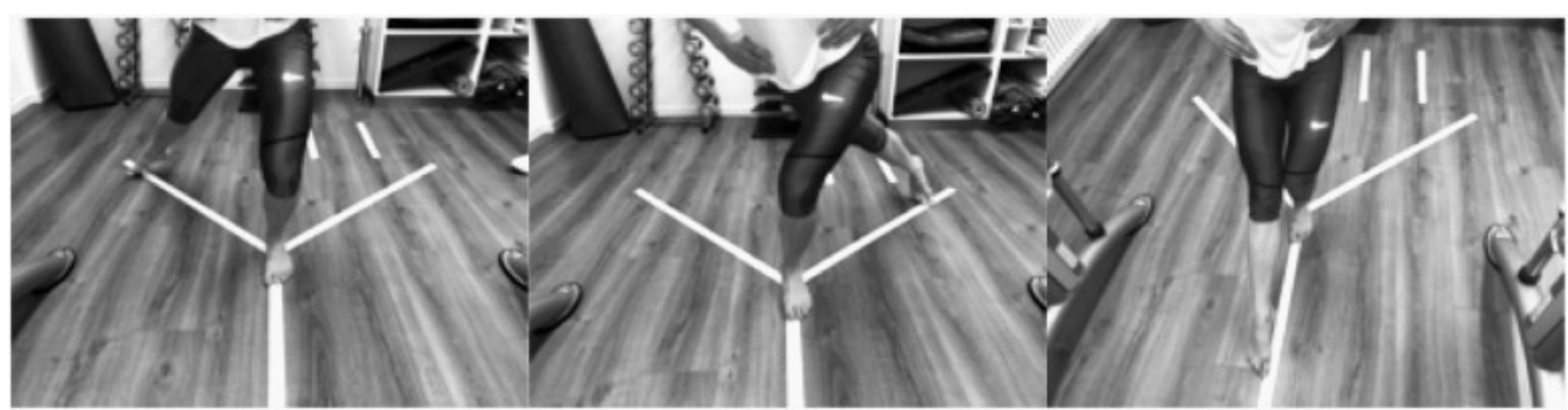

Abb. 16: Y-Balance-Test. Test zur posturalen Kontrolle/Sensomotorik. Ein Bein steht im Zentrum des Y. Mit dem anderen Fuss (Grosszehe) muss in möglichst grossem Abstand zum Zentrum der Boden berührt werden ohne das Gleichgewicht zu verlieren. 


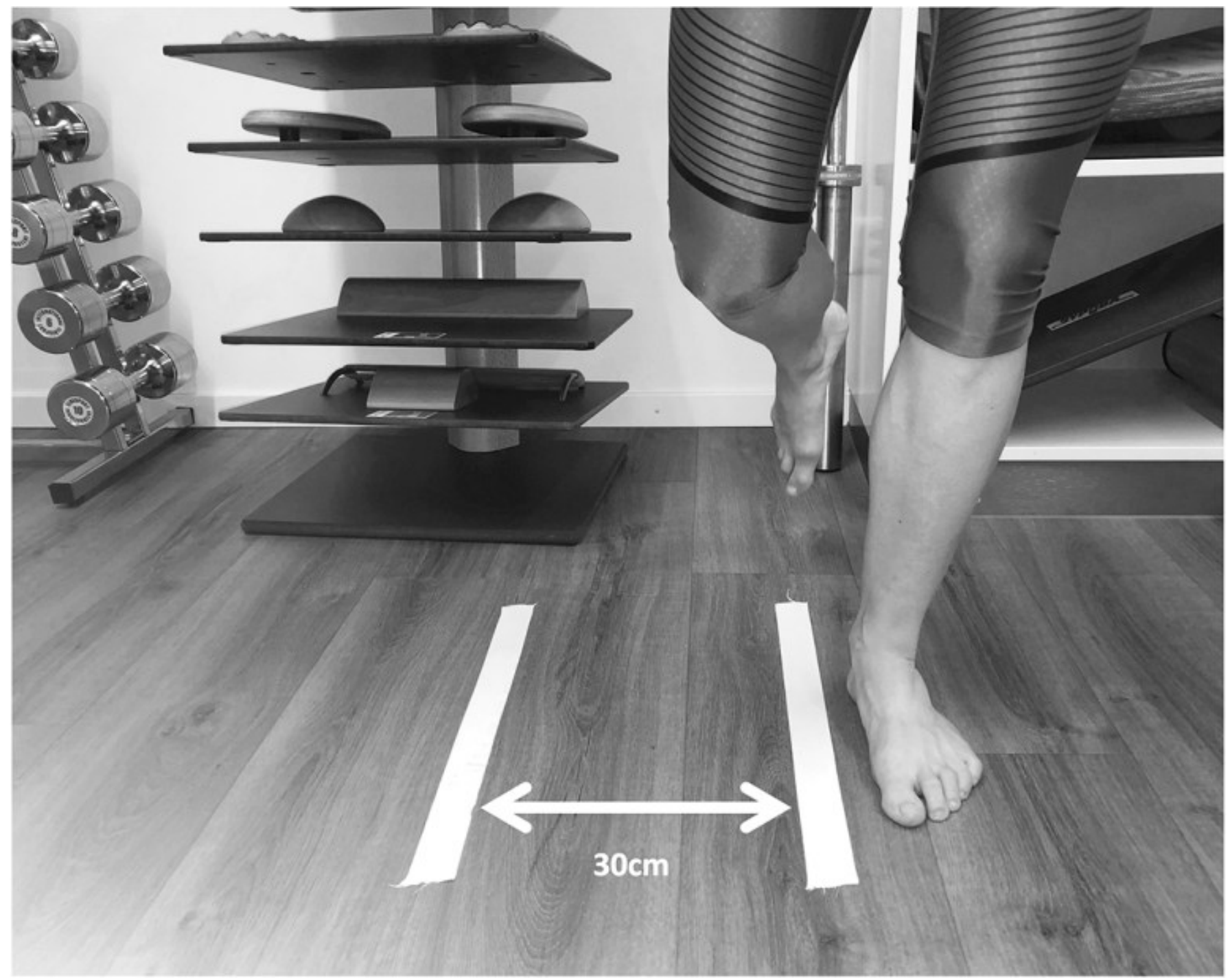

Abb. 17: Side hop Test. Ein minimaler Abstand von $30 \mathrm{~cm}$ muss eingehalten werden. Dabei wird die Zeit für 10 Sprünge oder die Anzahl Sprünge über 30 Sekunden gemessen.

\section{Dynamische Tests}

Die dynamische Untersuchung kann einfache, globale Tests bis hin zu objektivierbaren funktionellen Tests verschiedene Komplexitätsstufen beinhalten.

Einfache, globale Tests beinhalten die Analyse des Gangbildes (dynamische Varus-/Valgusfehlbelastungen, Rotationsbewegungen (z.B. Giving-way), die Beinachsen- und Beckenstabilität im Gang, sowie das Gehen im Zehenspitzen- und Fersengang.

Eine Laufanalyse kann das Laufbild analysieren. Hierzu haben sich heutzutage instrumentierte Laufanalysen (zwei- oder dreidimensional) mit Kameras und Markern etabliert. Eine Sprunganalyse (z.B. einbeinige Seitwärtssprünge oder Laufsprünge) kann subtilere Becken- und Beinachseninstabilitäten detektieren als eine normale Ganganalyse.

Verschiedene, einfache funktionelle Tests können jedoch in eine klinische Prüfung miteinbezogen werden. Es werden unzählige Tests beschrieben. Allen gemeinsam ist, dass sie eine globale Prüfung der sensomotorischen Kontrolle darstellen und durch eine globale funktionelle Insuffizienz oder eine isolierte 
Pathologie (z.B. Kniegelenkserguss) beeinträchtig sein können. Sie können in der Diagnostik sowohl im Seitenvergleich als auch in einem repetitiven Setting zum Monitoring eines Trainingseffekts herangezogen werden. Das Bedürfnis nach funktionellen Tests richtet sich stark nach der Sportart und ihrem Anforderungsprofil. Zwei oft verwendete funktionelle Tests sind:

(I) DStar Excursion Balance Test (SEBT), auch Y-Balance Test als vereinfachte Form (Abb. 16). Testung der sensomotorischen Balance. Im Einbeinstand wird mit dem anderen Bein auf drei (Y) oder 8 (SEBT)Strecken die maximale Länge gemessen, bei der die Balance gehalten werden kann.

(II) $\square$ Side Hop Test (Abb. 17): Über eine Distanz von $30 \mathrm{~cm}$ muss einbeinig seitwärts gehüpft werden. Gezählt werden entweder die Anzahl Sprünge in 30 Sekunden oder die Zeit für 10 Sprünge. Die Seitendifferenz darf nicht mehr als 10\% betragen.

Auswertebögen können für beide Tests frei im Internet heruntergeladen werden, z.B. auf https://www.sart.ch/infocenter/download/

\section{Korrespondenzadresse}

PD Dr. med. Dr. phil. André Leumann

OrthoPraxis Leumann

Claragraben 78

4058 Basel

E-Mail: info@orthopraxisleumann.ch

Tel. +41616926900

Fax +41616839292ロ

\section{Referenzen}

1. AGA Komitee Hüfte. Diagnostik des Hüftgelenks. 2017. Aga-online.de Buckup K. Clinical Tests for the Musculoskeletal System. Examinations - Signs - Phenomena. Thieme, Stuttgart und New York, 2004, 1-272.

2. Fromm L, Meyer P, Vavken P, Leumann A. Outcome von Muskelverletzungen im Nachwuchsfussball. SEM. 2018;67:28-32.

3. Ockert B, Haasters F, Polzer H, Grote S, Kessler MA, Mutschler W, Kanz KG. Der verletzte Meniskus: Wie sicher ist die klinische Untersuchung? Eine Metaanalyse. Unfallchirurg. 2010;113:293-9.

4. https://www.sart.ch/infocenter/download/

5. https://sgsm.ch/publikationen/spu-dokumente

6. https://sgsm.ch/weiter-fortbildung/weiterbildung/logbook

7. https://www.unfallstatistik.ch/d/publik/unfstat/pdf/Ts18.pdf 
CLINICAL EXAMINATION CRUCIATE LIGAMENT FOOT AND ANKLE HIP KNEE MUSCLE RANGE OF MOTION 